\title{
Quantum Teichmüller space and Kashaev algebra
}

\author{
REN GuO \\ XIAOBO LiU
}

\begin{abstract}
Kashaev algebra associated to a surface is a noncommutative deformation of the algebra of rational functions of Kashaev coordinates. For two arbitrary complex numbers, there is a generalized Kashaev algebra. The relationship between the shear coordinates and Kashaev coordinates induces a natural relationship between the quantum Teichmüller space and the generalized Kashaev algebra.
\end{abstract}

57R56; 57M50, 20G42

\section{Introduction}

A quantization of the Teichmüller space $\mathcal{T}(S)$ of a punctured surface $S$ was developed by Chekhov and Fock $[6 ; 7 ; 8]$ and, independently, by Kashaev $[9 ; 10 ; 11 ; 12]$. This is a deformation of the $\mathrm{C}^{*}$-algebra of functions on Teichmüller space $\mathcal{T}(S)$. The quantization was expressed in terms of self-adjoint operators on Hilbert spaces and the quantum dilogarithm function. Although these two approaches of quantization use the same ingredients, the relationship between them is still mysterious. Chekhov and Fock worked with shear coordinates of Teichmüller space while Kashaev worked with a new coordinate.

The pure algebraic foundation of Chekhov-Fock's quantization was established by the second author [14] (see also work with Bonahon and Bai [5; 2]). In this paper we investigate the algebraic aspect of Kashaev's quantization and establish a natural relationship between these two algebraic theories. This algebraic relationship should shed light on the two approach of operator-theoritical quantization of Teichmüller space.

\subsection{Quantum Teichmüller space}

Let's review the finite dimensional Chekhov-Fock's quantization following [14]. Let $S$ be an oreinted surface of finite topological type, with genus $g$ and with $p \geq 1$ punctures, obtained by removing $p$ points $\left\{v_{1}, \ldots, v_{p}\right\}$ from a closed oriented surface $\bar{S}$ of genus $g$. If the Euler characteristic of $S$ is negative, that is, $m:=2 g-2+p>0$, 
$S$ admits complete hyperbolic metrics. The Teichmüller space $\mathcal{T}(S)$ of $S$ consists of all isotopy classes of complete hyperbolic metrics on $S$.

An ideal triangulation of $S$ is a triangulation of the closed surface $\bar{S}$ whose vertex set is exactly $\left\{v_{1}, \ldots, v_{p}\right\}$. Under a complete hyperbolic metric, an ideal triangulation of $S$ is realized as a proper 1-dimensional submanifold whose complementary regions are hyperbolic ideal triangles. William Thurston [17] associated to each ideal triangulation a global coordinate system which is called shear coordinate (see also Bonahon [3] and Fock [7]). Given two ideal triangulations $\lambda$ and $\lambda^{\prime}$, the corresponding coordinate changes are rational, so that there is a well-defined notion of rational functions on $\mathcal{T}(S)$.

For an ideal triangulation $\lambda$ and a number $q=\mathrm{e}^{\pi \mathrm{i} \hbar} \in \mathbb{C}$, the Chekhov-Fock algebra $\mathcal{T}_{\lambda}^{q}$ is the algebra over $\mathbb{C}$ defined by generators $X_{1}^{ \pm 1}, X_{2}^{ \pm 1}, \ldots, X_{3 m}^{ \pm 1}$ associated to the components of $\lambda$ and by relations $X_{i} X_{j}=q^{2 \sigma_{i j}^{\lambda} X_{j} X_{i}}$, where the numbers $\sigma_{i j}^{\lambda}$ are integers determined by the combinatorics of the ideal triangulation $\lambda$. This algebra has a well-defined fraction division algebra $\widehat{\mathcal{T}}_{\lambda}^{q}$.

As one moves from one ideal triangulation $\lambda$ to another $\lambda^{\prime}$, Chekhov and Fock $[7 ; 8$; 6] (see also [14]) introduce coordinate change isomorphisms $\Phi_{\lambda \lambda^{\prime}}^{q}: \hat{\mathcal{T}}_{\lambda^{\prime}}^{q} \rightarrow \widehat{\mathcal{T}}_{\lambda}^{q}$ which satisfy the natural property that $\Phi_{\lambda^{\prime \prime} \lambda^{\prime}}^{q} \circ \Phi_{\lambda^{\prime} \lambda}^{q}=\Phi_{\lambda^{\prime \prime} \lambda}^{q}$ for any ideal triangulations $\lambda, \lambda^{\prime}$, $\lambda^{\prime \prime}$. In a triangulation independent way, this associates to the surface $S$ the algebra $\hat{\mathcal{T}}_{S}^{q}$ defined as the quotient of the family of all $\hat{\mathcal{T}}_{\lambda}^{q}$, with $\lambda$ ranging over ideal triangulations of the surface $S$, by the equivalence relation that identifies $\hat{\mathcal{T}}_{\lambda}^{q}$ and $\hat{\mathcal{T}}_{\lambda^{\prime}}^{q}$ by the coordinate change isomorphism $\Phi_{\lambda \lambda^{\prime}}^{q}$. The algebra $\hat{\mathcal{T}}_{S}^{q}$ is called the quantum Teichmüller space of the surface $S$. It turns out that $\Phi_{\lambda \lambda^{\prime}}^{1}$ is just the corresponding shear coordinate changes. Therefore, the quantum Teichmüller space $\hat{\mathcal{T}}_{S}^{q}$ is a noncommutative deformation of the algebra of rational functions on the Teichmüller space $\mathcal{T}(S)$.

\subsection{Generalized Kashaev algebra}

A decorated ideal triangulation of a punctured surface $S$ is an ideal triangulation such that the ideal triangles are numerated and there is a mark at a corner of each triangle. Kashaev [9] introduced a new coordinate associated to a decorated ideal triangulation of $S$. A Kashaev coordinate associated to a decorated ideal triangulation is a vector in $\mathbb{R}^{4 m}$ which assigns two numbers to a decorated ideal triangle. For two decorated ideal triangulation $\tau$ and $\tau^{\prime}$ the corresponding coordinate changes are rational.

For a decorated ideal triangulation $\tau$ and a number $q=\mathrm{e}^{\pi \mathrm{i} \hbar} \in \mathbb{C}$, Kashaev introduced an algebra $\mathcal{K}_{\tau}^{q}$ which is the algebra over $\mathbb{C}$ defined by generators $Y_{1}^{ \pm 1}, Z_{1}^{ \pm 1}, \ldots$, 
$Y_{2 m}^{ \pm 1}, Z_{2 m}^{ \pm 1}$ associated to ideal triangles of $\tau$ and by relations

$$
\begin{aligned}
Y_{i} Y_{j} & =Y_{j} Y_{i}, & Z_{i} Z_{j} & =Z_{j} Z_{i}, \\
Y_{i} Z_{j} & =Z_{j} Y_{i} \text { if } i \neq j, & Z_{i} Y_{i} & =q^{2} Y_{i} Z_{i} .
\end{aligned}
$$

Let $\widehat{\mathcal{K}}_{\tau}^{q}$ be the fraction division algebra of $\mathcal{K}_{\tau}^{q}$.

As one moves from one decorated ideal triangulation $\tau$ to another $\tau^{\prime}$, Kashaev [9] introduced coordinate change isomorphisms from $\widehat{\mathcal{K}}_{\tau^{\prime}}^{q}$ to $\widehat{\mathcal{K}}_{\tau}^{q}$. Analog to the construction of quantum Teichmüller space, there is an algebra $\hat{\mathcal{K}}_{S}^{q}$ associated to a surface which is independent of decorated ideal triangulations.

In this paper, we will show Kashaev's construction of coordinate change isomorphisms are not unique. In fact, for two arbitrary complex numbers $a, b$, there are coordinate change isomorphisms from $\widehat{\mathcal{K}}_{\tau^{\prime}}^{q}$ to $\widehat{\mathcal{K}}_{\tau}^{q}$. And Kashaev's construction is the special case of $a=q^{-1}, b=q$. For this generalized coordinate change isomorphisms, we also obtain a well-defined noncommutative algebra $\widehat{\mathcal{K}}_{S}^{q}(a, b)$ associated to the surface $S$ which is called the generalized Kashaev algebra. This is stated in Theorem 8.

\subsection{The relationship between quantum Teichmüller space and Kashaev algebra}

To understand the relationship between the quantum Teichmüller space and Kashaev algebra, we need to first understand the relationship between shear coordinates and Kashaev coordinates. Fix a decorated ideal triangulation, the space of Kashaev coordinates is a fiber bundle on a subset in the enhanced Teichmüller space whose fiber is an affine space modeled on the homology group $H_{1}(S, \mathbb{R})$. This is proved in Theorem 9.

The relationship between the shear coordinates and Kashaev coordinates induces a natural relationship between the quantum Teichmüller space $\hat{\mathcal{T}}_{S}^{q}$ and the generalized Kashaev algebra $\widehat{\mathcal{K}}_{S}^{q}(a, b)$. We show that there is a homomorphism from the quotient algebra $\hat{\mathcal{T}}_{S}^{q} /\left(q^{-2 m-\sum_{i<j} \sigma_{i j}^{\lambda}} X_{1} X_{2} \ldots X_{3 m}\right)$ to $\hat{\mathcal{K}}_{S}^{q}(a, b)$ if and only if $a=q^{-2}$ and $b=q^{3}$. This is proved in Corollary 19 and Theorem 20. The result explains why we need to look for new construction of coordinate change isomorphisms other than Kashaev's construction.

\subsection{Open questions}

Hua Bai [1] proved that the construction of quantum Teichmüller space $\hat{\mathcal{T}}_{S}^{q}$ is essentially unique. The uniqueness of the algebra $\widehat{\mathcal{K}}_{S}^{q}(a, b)$ should be an interesting problem. 
In work by Bonahon and the second author $[5 ; 13 ; 4]$, it is shown that quantum Teichmüller space $\hat{\mathcal{T}}_{S}^{q}$ has a rich representation theory which also produces an invariant of hyperbolic 3-manifolds. The representation theory of the algebra $\hat{\mathcal{K}}_{S}^{q}(a, b)$ should be investigated.

One of the motivation of this paper is to understand the relationship between ChekhovFock and Kashaev's operator-theoretical quantization. It is important to find a relationship between the two operator algebras involving the quantum dilogarithm function.

\section{Decorated ideal triangulations}

Let $S$ be an oriented surface of genus $g$ with $p \geq 1$ punctures and negative Euler characteristic, that is, $m=2 g-2+p>0$. Any ideal triangulation has $2 m$ ideal triangles and $3 m$ edges.

A decorated ideal triangulation $\tau$ of $S$ was introduced by Kashaev [9] as an ideal triangulation such that the ideal triangles are numerated as $\left\{\tau_{1}, \tau_{2}, \ldots, \tau_{2 m}\right\}$ and there is a mark (a star symbol) at a corner of each ideal triangle. Denote by $\triangle(S)$ the set of isotopy classes of decorated ideal triangulations of the surface $S$.

The set $\triangle(S)$ admits a natural action of the group $\mathfrak{S}_{2 m}$ of permutations of $2 m$ elements, acting by permuting the indexes of the ideal triangles of $\tau$. Namely $\tau^{\prime}=\alpha(\tau)$ for $\alpha \in \mathfrak{S}_{2 m}$ if its $i$-th ideal triangle $\tau_{i}^{\prime}$ is equal to $\tau_{\alpha(i)}$.

Another important transformation of $\triangle(S)$ is provided by the diagonal exchange $\varphi_{i j}: \triangle(S) \rightarrow \triangle(S)$ defined as follows. Suppose that two ideal triangles $\tau_{i}, \tau_{j}$ share an edge $e$ such that the marked corners are opposite to the edge $e$. Then $\varphi_{i j}(\tau)$ is obtained by rotating the interior of the union $\tau_{i} \cup \tau_{j} 90^{\circ}$ in the clockwise order, as illustrated in Figure 1(2).

The last one of transformations of $\triangle(S)$ is the mark rotation $\rho_{i}: \Delta(S) \rightarrow \triangle(S)$. $\rho_{i}(\tau)$ is obtained by relocating the mark of the ideal triangle $\tau_{i}$ from one corner to the next corner in the counterclockwise order, as illustrated in Figure 1(1).

Lemma 1 The reindexings, diagonal exchanges and mark rotations satisfy the following relations:

(1) $(\alpha \beta)(\tau)=\alpha(\beta(\tau))$ for every $\alpha, \beta \in \mathfrak{S}_{2 m}$;

(2) $\varphi_{i j} \circ \varphi_{i j}=\alpha_{i \leftrightarrow j}$, where $\alpha_{i \leftrightarrow j}$ denotes the transposition exchanging $i$ and $j$;

(3) $\alpha \circ \varphi_{i j}=\varphi_{\alpha(i) \alpha(j)} \circ \alpha$ for every $\alpha \in \mathfrak{S}_{2 m}$;

(4) $\varphi_{i j} \circ \varphi_{k l}(\tau)=\varphi_{k l} \circ \varphi_{i j}(\tau)$, for $\{i, j\} \neq\{k, l\}$; 

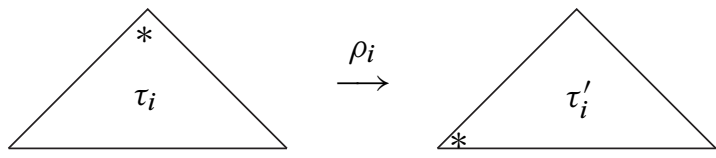

(1)
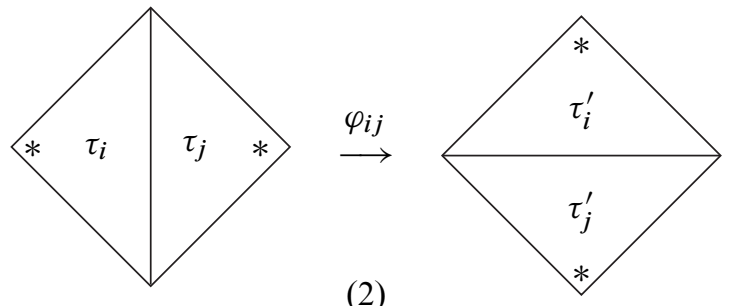

(2)

Figure 1

(5) If three triangles $\tau_{i}, \tau_{j}, \tau_{k}$ of an ideal triangulation $\tau \in \Delta(S)$ form a pentagon and their marked corners are located as in Figure 2, then the Pentagon Relation

$$
\omega_{j k} \circ \omega_{i k} \circ \omega_{i j}(\tau)=\omega_{i j} \circ \omega_{j k}(\tau)
$$

holds, where $\omega_{\mu \nu}=\rho_{\mu} \circ \varphi_{\mu \nu} \circ \rho_{\nu}$;

(6) $\rho_{i} \circ \rho_{i} \circ \rho_{i}=\mathrm{Id}$;

(7) $\rho_{i} \circ \rho_{j}=\rho_{j} \circ \rho_{i}$;

(8) $\alpha \circ \rho_{i}=\rho_{i} \circ \alpha$ for for every $\alpha \in \mathfrak{S}_{2 m}$.

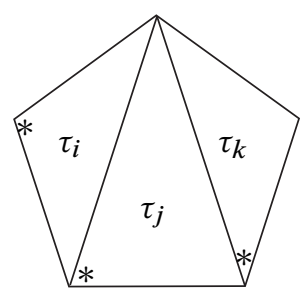

Figure 2

The lemma can be proved by drawing graphs.

Remark Lemma 1 is essentially contained in Kashaev [10] where $\omega_{i j}$ is used as the diagonal exchange.

The following two results about decorated ideal triangulations can be easily proved using Penner's result about ideal triangulations [15]. 
Theorem 2 Given two decorated ideal triangulations $\tau, \tau^{\prime} \in \triangle(S)$, there exists a finite sequence of decorated ideal triangulations $\tau=\tau_{(0)}, \tau_{(1)}, \ldots, \tau_{(n)}=\tau^{\prime}$ such that each $\tau_{(k+1)}$ is obtained from $\tau_{(k)}$ by a diagonal exchange or by a mark rotation or by a reindexing of its ideal triangles.

Theorem 3 Given two decorated ideal triangulations $\tau, \tau^{\prime} \in \triangle(S)$ and given two sequences $\tau=\tau_{(0)}, \tau_{(1)}, \ldots, \tau_{(n)}=\tau^{\prime}$ and $\tau=\bar{\tau}_{(0)}, \bar{\tau}_{(1)}, \ldots, \bar{\tau}_{(\bar{n})}=\tau^{\prime}$ of diagonal exchanges, mark rotations and reindexings connecting them as in Theorem 2, these two sequences can be related to each other by successive applications of the following moves and of their inverses. These moves correspond to the relations in Lemma 1.

(1) Replace $\ldots, \tau_{(k)}, \beta\left(\tau_{(k)}\right), \alpha \circ \beta\left(\tau_{(k)}\right), \ldots$

by $\ldots, \tau_{(k)},(\alpha \beta)\left(\tau_{(k)}\right), \ldots$ where $\alpha, \beta \in \mathfrak{S}_{n}$.

(2) Replace $\ldots, \tau_{(k)}, \varphi_{i j}\left(\tau_{(k)}\right), \varphi_{i j} \circ \varphi_{i j}\left(\tau_{(k)}\right) \ldots$

by $\ldots, \tau_{(k)}, \alpha_{i \leftrightarrow j}\left(\tau_{(k)}\right), \ldots$.

(3) Replace $\ldots, \tau_{(k)}, \varphi_{i j}\left(\tau_{(k)}\right), \alpha \circ \varphi_{i j}\left(\tau_{(k)}\right), \ldots$

by $\ldots, \tau_{(k)}, \alpha\left(\tau_{(k)}\right), \varphi_{\alpha(i) \alpha(j)} \circ \alpha\left(\tau_{(k)}\right), \ldots$ where $\alpha \in \mathfrak{S}_{n}$.

(4) Replace $\ldots, \tau_{(k)}, \varphi_{k l}\left(\tau_{(k)}\right), \varphi_{i j} \circ \varphi_{k l}\left(\tau_{(k)}\right), \ldots$

by $\ldots, \tau_{(k)}, \varphi_{i j}\left(\tau_{(k)}\right), \varphi_{k l} \circ \varphi_{i j}\left(\tau_{(k)}\right), \ldots$ where $\{i, j\} \neq\{k, l\}$.

(5) Replace ..., $\tau_{(k)}, \omega_{i j}\left(\tau_{(k)}\right), \omega_{i k} \circ \omega_{i j}\left(\tau_{(k)}\right), \omega_{j k} \circ \omega_{i k} \circ \omega_{i j}\left(\tau_{(k)}\right), \ldots$,

by $\ldots, \tau_{(k)}, \omega_{j k}\left(\tau_{(k)}\right), \omega_{i j} \circ \omega_{j k}\left(\tau_{(k)}\right), \ldots$ where $\omega_{\mu \nu}=\rho_{\mu} \circ \varphi_{\mu \nu} \circ \rho_{\nu}$.

(6) Replace .., $\tau_{(k)}, \rho_{i}\left(\tau_{(k)}\right), \rho_{i} \circ \rho_{i}\left(\tau_{(k)}\right), \tau_{(k)} \ldots$

by $\ldots, \tau_{(k)}, \ldots$.

(7) Replace .., $\tau_{(k)}, \rho_{i}\left(\tau_{(k)}\right), \rho_{j} \circ \rho_{i}\left(\tau_{(k)}\right), \ldots$

by $\ldots, \tau_{(k)}, \rho_{j}\left(\tau_{(k)}\right), \rho_{i} \circ \rho_{j}\left(\tau_{(k)}\right), \ldots$.

(8) Replace .., $\tau_{(k)}, \rho_{i}\left(\tau_{(k)}\right), \alpha \circ \rho_{i}\left(\tau_{(k)}\right), \ldots$

by $\ldots, \tau_{(k)}, \alpha\left(\tau_{(k)}\right), \rho_{i} \circ \alpha\left(\tau_{(k)}\right), \ldots$.

\section{Generalized Kashaev algebra}

For a decorated ideal triangulation $\tau$ of a punctured surface $S$, Kashaev [9] associated each ideal triangle $\tau_{i}$ two numbers $\ln y_{i}, \ln z_{i}$. A Kashaev coordinate is a vector $\left(\ln y_{1}, \ln z_{1}, \ldots, \ln y_{2 m}, \ln z_{2 m}\right) \in \mathbb{R}^{4 m}$.

Denote by $\left(y_{1}, z_{1}, \ldots, y_{2 m}, z_{2 m}\right)$ the exponential Kashaev coordinate for the decorated ideal triangulation $\tau$. Denote by $\left(y_{1}^{\prime}, z_{1}^{\prime}, \ldots, y_{2 m}^{\prime}, z_{2 m}^{\prime}\right)$ the exponential Kashaev coordinate for the decorated ideal triangulation $\tau^{\prime}$. 
Definition 4 (Kashaev [9]) Suppose that a decorated ideal triangulation $\tau^{\prime}$ is obtained from another one $\tau$ by reindexing the ideal triangles, that is, $\tau^{\prime}=\alpha(\tau)$ for some $\alpha \in \mathfrak{S}_{2 m}$, then we define $\left(y_{i}^{\prime}, z_{i}^{\prime}\right)=\left(y_{\alpha(i)}, z_{\alpha(i)}\right)$ for any $i=1, \ldots, 2 m$.

Suppose that a decorated ideal triangulation $\tau^{\prime}$ is obtained from another one $\tau$ by a mark rotation, that is, $\tau^{\prime}=\rho_{i}(\tau)$ for some $i$, then we define $\left(y_{j}^{\prime}, z_{j}^{\prime}\right)=\left(y_{j}, z_{j}\right)$ for any $j \neq i$ while

$$
\left(y_{i}^{\prime}, z_{i}^{\prime}\right)=\left(\frac{z_{i}}{y_{i}}, \frac{1}{y_{i}}\right) .
$$

Suppose a decorated ideal triangulation $\tau^{\prime}$ is obtained from another one $\tau$ by a diagonal exchange, that is, $\tau^{\prime}=\varphi_{i j}(\tau)$ for some $i, j$, then we define $\left(y_{k}^{\prime}, z_{k}^{\prime}\right)=\left(y_{k}, z_{k}\right)$ for any $k \notin\{i, j\}$ while

$$
\left(y_{i}^{\prime}, z_{i}^{\prime}, y_{j}^{\prime}, z_{j}^{\prime}\right)=\left(\frac{z_{j}}{y_{i} y_{j}+z_{i} z_{j}}, \frac{y_{i}}{y_{i} y_{j}+z_{i} z_{j}}, \frac{z_{i}}{y_{i} y_{j}+z_{i} z_{j}}, \frac{y_{j}}{y_{i} y_{j}+z_{i} z_{j}}\right) .
$$

Remark Kashaev [9] considered $\omega_{i j}$ instead of $\varphi_{i j}$.

There is a natural relationship between Kashaev coordinates and Penner coordinates which is established in [9]. For an exposition, see also Teschner [16]. In the Appendix of this paper, we include the main feature of this topic. Especially, the changes of Kashaev coordinate in Definition 4 are compatible with the changes of Penner coordinates.

For a decorated ideal triangulation $\tau$ of a punctured surface $S$, Kashaev [9] introduced an algebra $\mathcal{K}_{\tau}^{q}$ on $\mathbb{C}$ generated by $Y_{1}^{ \pm}, Z_{1}^{ \pm}, Y_{2}^{ \pm}, Z_{2}^{ \pm}, \ldots, Y_{2 m}^{ \pm}, Z_{2 m}^{ \pm}$, with $Y_{i}^{ \pm}, Z_{i}^{ \pm}$ associated to an ideal triangle $\tau_{i}$, and by the relations (1):

$$
\begin{aligned}
Y_{i} Y_{j} & =Y_{j} Y_{i}, & Z_{i} Z_{j} & =Z_{j} Z_{i}, \\
Y_{i} Z_{j} & =Z_{j} Y_{i} \text { if } i \neq j, & Z_{i} Y_{i} & =q^{2} Y_{i} Z_{i}
\end{aligned}
$$

Remark Kashaev's original definition is $Y_{i} Z_{i}=q^{2} Z_{i} Y_{i}$. We adopt our convention to make it compatible with the quantum Teichmüller space [14]. Kashaev's parameter $q$ corresponds to our $q^{-1}$.

The algebra $\widehat{\mathcal{K}}_{\tau}^{q}$ is the fraction division algebra of $\mathcal{K}_{\tau}^{q}$ which consists of all the factors $F G^{-1}$ with $F, G \in \mathcal{K}_{\tau}^{q}$ and $Q \neq 0$, and two such fractions $F_{1} G_{1}^{-1}$ and $F_{2} G_{2}^{-1}$ are identified if there exists $S_{1}, S_{2} \in \mathcal{K}_{\tau}^{q}-\{0\}$ such that $P_{1} S_{1}=P_{2} S_{2}$ and $Q_{1} S_{1}=Q_{2} S_{2}$. In particular, when $q=1, \mathcal{K}_{\tau}^{q}$ and $\widehat{\mathcal{K}}_{\tau}^{q}$ respectively coincide with the Laurent polynomial algebra $\mathbb{C}\left[Y_{1}^{ \pm}, Z_{1}^{ \pm}, \ldots, Y_{2 m}^{ \pm}, Z_{2 m}^{ \pm}\right]$and with the rational fraction algebra $\mathbb{C}\left(Y_{1}, Z_{1}, \ldots, Y_{2 m}, Z_{2 m}\right)$. The general $\mathcal{K}_{\tau}^{q}$ and $\widehat{\mathcal{K}}_{\tau}^{q}$ can be considered as deformations of $\mathcal{K}_{\tau}^{1}$ and $\hat{\mathcal{K}}_{\tau}^{1}$. 
The algebra $\widehat{\mathcal{K}}_{\tau}^{q}$ depends on the decorated ideal triangulation $\tau$. We introduce algebra isomorphisms in the following.

Definition 5 For any numbers $a, b \in \mathbb{C}$.

Suppose that a decorated ideal triangulation $\tau^{\prime}$ is obtained from another one $\tau$ by reindexing the ideal triangles, that is, $\tau^{\prime}=\alpha(\tau)$ for some $\alpha \in \mathfrak{S}_{2 m}$, then we define a map $\hat{\alpha}: \widehat{\mathcal{K}}_{\tau^{\prime}}^{q} \rightarrow \widehat{\mathcal{K}}_{\tau}^{q}$ by indicating the image of generators and extend it to the whole algebra:

$$
\begin{aligned}
& \hat{\alpha}\left(Y_{i}^{\prime}\right)=Y_{\alpha(i)}, \text { for any } i=1, \ldots, 2 m, \\
& \hat{\alpha}\left(Z_{i}^{\prime}\right)=Z_{\alpha(i)}, \text { for any } i=1, \ldots, 2 m .
\end{aligned}
$$

Suppose that a decorated ideal triangulation $\tau^{\prime}$ is obtained from another one $\tau$ by a mark rotation, that is, $\tau^{\prime}=\rho_{i}(\tau)$ for some $i$, then we define a map $\hat{\rho}_{i}: \widehat{\mathcal{K}}_{\tau^{\prime}}^{q} \rightarrow \widehat{\mathcal{K}}_{\tau}^{q}$ by indicating the image of generators and extend it to the whole algebra:

$$
\begin{array}{ll}
\hat{\rho}_{i}\left(Y_{j}^{\prime}\right)=Y_{j}, \text { if } j \neq i, & \hat{\rho}_{i}\left(Z_{j}^{\prime}\right)=Z_{j}, \text { if } j \neq i, \\
\hat{\rho}_{i}\left(Y_{i}^{\prime}\right)=a Y_{i}^{-1} Z_{i}, & \hat{\rho}_{i}\left(Z_{i}^{\prime}\right)=Y_{i}^{-1} .
\end{array}
$$

Suppose a decorated ideal triangulation $\tau^{\prime}$ is obtained from another one $\tau$ by a diagonal exchange, that is, $\tau^{\prime}=\varphi_{i j}(\tau)$ for some $i, j$, then we define a map $\hat{\varphi}_{i j}: \widehat{\mathcal{K}}_{\tau^{\prime}}^{q} \rightarrow \widehat{\mathcal{K}}_{\tau}^{q}$ by indicating the image of generators and extend it to the whole algebra:

$$
\begin{array}{ll}
\hat{\varphi}_{i j}\left(Y_{i}^{\prime}\right)=\left(b Y_{i} Y_{j}+Z_{i} Z_{j}\right)^{-1} Z_{j}, & \hat{\varphi}_{i j}\left(Z_{i}^{\prime}\right)=b\left(b Y_{i} Y_{j}+Z_{i} Z_{j}\right)^{-1} Y_{i}, \\
\hat{\varphi}_{i j}\left(Y_{j}^{\prime}\right)=\left(b Y_{i} Y_{j}+Z_{i} Z_{j}\right)^{-1} Z_{i}, & \hat{\varphi}_{i j}\left(Z_{j}^{\prime}\right)=b\left(b Y_{i} Y_{j}+Z_{i} Z_{j}\right)^{-1} Y_{j} .
\end{array}
$$

Remark From the definition, when $a=b=1$, we get the coordinate change formula in Definition 4.

Remark Kashaev [9] considered a special case of these maps when $a=q^{-1}, b=q$.

Proposition 6 The maps $\hat{\alpha}, \hat{\rho}_{i}$ and $\hat{\varphi}_{i j}$ satisfy the following relations which correspond to the relations in Lemma 1:

(1) $\widehat{\alpha \beta}=\widehat{\alpha} \circ \widehat{\beta}$ for every $\alpha, \beta \in \mathfrak{S}_{2 m}$;

(2) $\hat{\varphi}_{i j} \circ \hat{\varphi}_{i j}=\widehat{\alpha}_{i \leftrightarrow j}$;

(3) $\hat{\alpha} \circ \hat{\varphi}_{i j}=\hat{\varphi}_{\alpha(i) \alpha(j)} \circ \hat{\alpha}$ for every $\alpha \in \mathfrak{S}_{2 m}$;

(4) $\hat{\varphi}_{i j} \circ \widehat{\varphi}_{k l}=\hat{\varphi}_{k l} \circ \hat{\varphi}_{i j}$ for $\{i, j\} \neq\{k, l\}$; 
(5) If three triangles $\tau_{i}, \tau_{j}, \tau_{k}$ of an ideal triangulation $\tau \in \Delta(S)$ form a pentagon and their marked corners are located as in Figure 3, then the Pentagon Relation holds:

$$
\widehat{\omega}_{j k} \circ \widehat{\omega}_{i k} \circ \widehat{\omega}_{i j}=\widehat{\omega}_{i j} \circ \widehat{\omega}_{j k},
$$

where $\hat{\omega}_{\mu \nu}=\hat{\rho}_{\mu} \circ \hat{\varphi}_{\mu \nu} \circ \hat{\rho}_{\nu}$;

(6) $\hat{\rho}_{i} \circ \hat{\rho}_{i} \circ \hat{\rho}_{i}=\mathrm{Id}$;

(7) $\hat{\rho}_{i} \circ \hat{\rho}_{j}=\hat{\rho}_{j} \circ \hat{\rho}_{i}$;

(8) $\hat{\alpha} \circ \hat{\rho}_{i}=\hat{\rho}_{i} \circ \hat{\alpha}$ for every $\alpha \in \mathfrak{S}_{2 m}$.

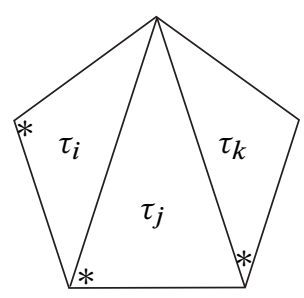

Figure 3: (Same as Figure 2)

Proof (1), (3), (4), (7), (8) are obvious.

(6) can be proved by using definition of $\hat{\rho}_{i}$ easily. In fact, we assume that

$$
\tau \stackrel{\rho_{i}}{\longleftarrow} \tau^{\prime \prime} \stackrel{\rho_{i}}{\longleftarrow} \tau^{\prime} \stackrel{\rho_{i}}{\longleftarrow} \tau \text {. }
$$

Then we have

$$
\widehat{\mathcal{K}}_{\tau}^{q} \stackrel{\hat{\rho}_{i}}{\rightarrow} \widehat{\mathcal{K}}_{\tau^{\prime \prime}}^{q} \stackrel{\hat{\rho}_{i}}{\rightarrow} \hat{\mathcal{K}}_{\tau^{\prime}}^{q} \stackrel{\hat{\rho}_{i}}{\rightarrow} \widehat{\mathcal{K}}_{\tau}^{q} .
$$

To show (6) is true, we need to show that $\hat{\rho}_{i} \circ \hat{\rho}_{i} \circ \hat{\rho}_{i}$ sends each generator of $\hat{\mathcal{K}}_{\tau}^{q}$ to itself. This is true for $Y_{j}, Z_{j}, j \neq i$. We only need to take care of $Y_{i}, Z_{i}$. For example, we check that $\hat{\rho}_{i} \circ \hat{\rho}_{i} \circ \hat{\rho}_{i}\left(Y_{i}\right)=Y_{i}$. In fact,

$$
\begin{aligned}
Y_{i} & \stackrel{\hat{\rho}_{i}}{\rightarrow} a Y_{i}^{\prime \prime-1} Z_{i}^{\prime \prime} \\
& \stackrel{\hat{\rho}_{i}}{\longrightarrow} a \hat{\rho}_{i}\left(Y_{i}^{\prime \prime-1}\right) \hat{\rho}_{i}\left(Z_{i}^{\prime \prime}\right)=a\left(a Y_{i}^{\prime-1} Z_{i}^{\prime}\right)^{-1} Y_{i}^{\prime-1}=Z_{i}^{\prime-1} \\
& \stackrel{\hat{\rho}_{i}}{\rightarrow} \hat{\rho}_{i}\left(Z_{i}^{\prime}\right)^{-1}=Y_{i} .
\end{aligned}
$$

To prove (2), we assume that

$$
\tau \stackrel{\alpha_{i \leftrightarrow j}}{\longleftarrow} \tau^{\prime \prime} \stackrel{\varphi_{i j}}{\longleftarrow} \tau^{\prime} \stackrel{\varphi_{i j}}{\longleftarrow} \tau .
$$


Then we have

$$
\hat{\mathcal{K}}_{\tau}^{q} \stackrel{\hat{\alpha}_{i \leftrightarrow j}}{\longrightarrow} \hat{\mathcal{K}}_{\tau^{\prime \prime}}^{q} \stackrel{\hat{\varphi}_{i j}}{\longrightarrow} \hat{\mathcal{K}}_{\tau^{\prime}}^{q} \stackrel{\hat{\varphi}_{i j}}{\longrightarrow} \hat{\mathcal{K}}_{\tau}^{q}
$$

To show that $\hat{\varphi}_{i j} \circ \hat{\varphi}_{i j} \circ \hat{\alpha}_{i \leftrightarrow j}=\mathrm{Id}$, we need to show that it sends every generator of $\hat{\mathcal{K}}_{\tau}^{q}$ to itself. This is true for $Y_{k}, Z_{k}, k \notin\{i, j\}$. We only need to take care of $Y_{i}, Z_{i} . Y_{j}, Z_{j}$. For example, we check it for $Y_{i}$. In fact,

$$
\begin{aligned}
Y_{i} \stackrel{\hat{\alpha}_{i \leftrightarrow j}}{\longrightarrow} Y_{j}^{\prime \prime} & \\
\stackrel{\widehat{\varphi}_{i j}}{\longrightarrow} & \left(b Y_{i}^{\prime} Y_{j}^{\prime}+Z_{i}^{\prime} Z_{j}^{\prime}\right)^{-1} Z_{i}^{\prime}, \\
\stackrel{\hat{\varphi}_{i j}}{\longrightarrow} & b\left(b Y_{i} Y_{j}+Z_{i} Z_{j}\right)^{-1} Z_{j}\left(b Y_{i} Y_{j}+Z_{i} Z_{j}\right)^{-1} Z_{i}+ \\
& \left.b^{2}\left(b Y_{i} Y_{j}+Z_{i} Z_{j}\right)^{-1} Y_{i}\left(b Y_{i} Y_{j}+Z_{i} Z_{j}\right)^{-1} Y_{j}\right]^{-1} \widehat{\varphi}_{i j}\left(Z_{i}^{\prime}\right) \\
= & {\left[b\left(b Y_{i} Y_{j}+Z_{i} Z_{j}\right)^{-1}\left(b q^{2} Y_{i} Y_{j}+Z_{i} Z_{j}\right)^{-1} Z_{j} Z_{i}+\right.} \\
& \left.b^{2}\left(b Y_{i} Y_{j}+Z_{i} Z_{j}\right)^{-1}\left(b q^{2} Y_{i} Y_{j}+Z_{i} Z_{j}\right)^{-1} q^{2} Y_{i} Y_{j}\right]^{-1} \hat{\varphi}_{i j}\left(Z_{i}^{\prime}\right) \\
= & {\left[b\left(b Y_{i} Y_{j}+Z_{i} Z_{j}\right)^{-1}\left(b q^{2} Y_{i} Y_{j}+Z_{i} Z_{j}\right)^{-1}\left(Z_{j} Z_{i}+b q^{2} Y_{i} Y_{j}\right)\right]^{-1} \widehat{\varphi}_{i j}\left(Z_{i}^{\prime}\right) } \\
= & b^{-1}\left(b Y_{i} Y_{j}+Z_{i} Z_{j}\right) \hat{\varphi}_{i j}\left(Z_{i}^{\prime}\right) \\
= & b^{-1}\left(b Y_{i} Y_{j}+Z_{i} Z_{j}\right) b\left(b Y_{i} Y_{j}+Z_{i} Z_{j}\right)^{-1} Y_{i} \\
= & Y_{i} .
\end{aligned}
$$

To prove (4) the Pentagon Relation, we need more work. As stated in Lemma 1, the Pentagon Relation for the decorated ideal triangulation is

$$
\begin{array}{ll} 
& \omega_{j k} \circ \omega_{i k} \circ \omega_{i j}=\omega_{i j} \circ \omega_{j k} \\
\Longleftrightarrow \quad \rho_{j} \circ \varphi_{j k} \circ \rho_{k} \circ \rho_{i} \circ \varphi_{i k} \circ \rho_{k} \circ \rho_{i} \circ \varphi_{i j} \circ \rho_{j}=\rho_{i} \circ \varphi_{i j} \circ \rho_{j} \circ \rho_{j} \circ \varphi_{j k} \circ \rho_{k} \\
\Longleftrightarrow \quad \rho_{j} \circ \varphi_{j k} \circ \rho_{k} \circ \rho_{i} \circ \varphi_{i k} \circ \rho_{k} \circ \rho_{i} \circ \varphi_{i j} \circ \rho_{j} \circ \rho_{k}^{2} \circ \alpha_{j \leftrightarrow k} \circ \varphi_{j k} \\
\quad \circ \rho_{j} \circ \alpha_{i \leftrightarrow j} \circ \varphi_{i j} \circ \rho_{i}^{2}=\mathrm{Id},
\end{array}
$$

since $\rho_{i}^{-1}=\rho_{i}^{2}$ and $\varphi_{i j}^{-1}=\alpha_{i \leftrightarrow j} \circ \varphi_{i j}=\varphi_{i j} \circ \alpha_{i \leftrightarrow j}$. Assume that

$$
\begin{aligned}
& \tau \stackrel{\rho_{j}}{\longleftarrow} \tau^{(17)} \stackrel{\varphi_{j k}}{\longleftarrow} \tau^{(16)} \stackrel{\rho_{k}}{\longleftarrow} \tau^{(15)} \stackrel{\rho_{i}}{\longleftarrow} \tau^{(14)} \stackrel{\varphi_{i k}}{\longleftarrow} \tau^{(13)} \\
& \stackrel{\rho_{k}}{\longleftarrow} \tau^{(12)} \stackrel{\rho_{i}}{\longleftarrow} \tau^{(11)} \stackrel{\varphi_{i j}}{\longleftarrow} \tau^{(10)} \stackrel{\rho_{j}}{\longleftarrow} \tau^{(9)} \stackrel{\rho_{k}}{\longleftarrow} \tau^{(8)} \stackrel{\rho_{k}}{\longleftarrow} \tau^{(7)} \\
& \stackrel{\alpha_{j \leftrightarrow k}}{\longleftarrow} \tau^{(6)} \stackrel{\varphi_{j k}}{\longleftarrow} \tau^{(5)} \stackrel{\rho_{j}}{\longleftarrow} \tau^{(4)} \stackrel{\alpha_{i \leftrightarrow j}}{\longleftarrow} \tau^{(3)} \stackrel{\varphi_{i j}}{\longleftarrow} \tau^{(2)} \stackrel{\rho_{i}}{\longleftarrow} \tau^{(1)} \stackrel{\rho_{i}}{\longleftarrow} \tau
\end{aligned}
$$


Then we have

$$
\begin{aligned}
& \widehat{\mathcal{K}}_{\tau}^{q} \stackrel{\hat{\rho}_{j}}{\longrightarrow} \widehat{\mathcal{K}}_{\tau^{(17)}}^{q} \stackrel{\hat{\varphi}_{j k}}{\longrightarrow} \widehat{\mathcal{K}}_{\tau^{(16)}}^{q} \stackrel{\hat{\rho}_{k}}{\longrightarrow} \widehat{\mathcal{K}}_{\tau^{(15)}}^{q} \stackrel{\hat{\rho}_{i}}{\longrightarrow} \widehat{\mathcal{K}}_{\tau^{(14)}}^{q} \stackrel{\hat{\varphi}_{i k}}{\longrightarrow} \widehat{\mathcal{K}}_{\tau^{(13)}}^{q} \\
& \stackrel{\hat{\rho}_{k}}{\longrightarrow} \widehat{\mathcal{K}}_{\tau^{(12)}}^{q} \stackrel{\hat{\rho}_{i}}{\longrightarrow} \hat{\mathcal{K}}_{\tau^{(11)}}^{q} \stackrel{\hat{\varphi}_{i j}}{\longrightarrow} \hat{\mathcal{K}}_{\tau^{(10)}}^{q} \stackrel{\hat{\rho}_{j}}{\longrightarrow} \widehat{\mathcal{K}}_{\tau^{(9)}}^{q} \stackrel{\hat{\rho}_{k}}{\longrightarrow} \hat{\mathcal{K}}_{\tau^{(8)}}^{q} \stackrel{\hat{\rho}_{k}}{\longrightarrow} \hat{\mathcal{K}}_{\tau^{(7)}}^{q} \\
& \stackrel{\hat{\alpha}_{j \leftrightarrow k}}{\longrightarrow} \hat{\mathcal{K}}_{\tau^{(6)}}^{q} \stackrel{\hat{\varphi}_{j k}}{\longrightarrow} \hat{\mathcal{K}}_{\tau^{(5)}}^{q} \stackrel{\hat{\rho}_{j}}{\longrightarrow} \widehat{\mathcal{K}}_{\tau^{(4)}}^{q} \stackrel{\widehat{\alpha}_{i \leftrightarrow j}}{\longrightarrow} \widehat{\mathcal{K}}_{\tau^{(3)}}^{q} \stackrel{\hat{\varphi}_{i j}}{\longrightarrow} \widehat{\mathcal{K}}_{\tau^{(2)}}^{q} \stackrel{\hat{\rho}_{i}}{\longrightarrow} \widehat{\mathcal{K}}_{\tau^{(1)}}^{q} \stackrel{\hat{\rho}_{i}}{\longrightarrow} \widehat{\mathcal{K}}_{\tau}^{q} .
\end{aligned}
$$

To verify the Pentagon Relation, we need to show that the composition of maps sends every generator of $\hat{\mathcal{K}}_{\tau}^{q}$ to itself. This is true for $Y_{l}, Z_{l}, l \notin\{i, j, k\}$. We only need to take care of $Y_{i}, Z_{i}, Y_{j}, Z_{j}, Y_{k}, Z_{k}$. For example, we verify it holds for $Y_{i}$. For simplicity the notation, in the following calculation, we do not indicate the upper index of generators. For example, the second $Y_{i}$ in the following should be $Y_{i}^{(17)}$.

$$
\begin{aligned}
& Y_{i} \stackrel{\hat{\rho}_{j}}{\longrightarrow} Y_{i} \stackrel{\hat{\varphi}_{j k}}{\longrightarrow} Y_{i} \stackrel{\hat{\rho}_{k}}{\longrightarrow} Y_{i} \stackrel{\hat{\rho}_{i}}{\longrightarrow} a Y_{i}^{-1} Z_{i} \\
& \stackrel{\hat{\varphi}_{i k}}{\longrightarrow} a\left[\left(b Y_{i} Y_{k}+Z_{i} Z_{k}\right)^{-1} Z_{k}\right]^{-1} b\left(b Y_{i} Y_{k}+Z_{i} Z_{k}\right)^{-1} Y_{i}=a b Z_{k}^{-1} Y_{i} \\
& \quad \stackrel{\hat{\rho}_{k}}{\longrightarrow} a b Y_{k} Y_{i} \stackrel{\hat{\rho}_{i}}{\longrightarrow} a^{2} b Y_{k} Y_{i}^{-1} Z_{i} \\
& \quad \stackrel{\hat{\varphi}_{i j}}{\longrightarrow} a^{2} b Y_{k}\left[\left(b Y_{i} Y_{j}+Z_{i} Z_{j}\right)^{-1} Z_{j}\right]^{-1} b\left(b Y_{i} Y_{j}+Z_{i} Z_{j}\right)^{-1} Y_{i}=a^{2} b^{2} Y_{k} Z_{j}^{-1} Y_{i} \\
& \stackrel{\hat{\rho}_{j}}{\longrightarrow} a^{2} b^{2} Y_{k} Y_{j} Y_{i} \stackrel{\hat{\rho}_{k}}{\longrightarrow} a^{3} b^{2} Y_{k}^{-1} Z_{k} Y_{j} Y_{i} \stackrel{\hat{\rho}_{k}}{\longrightarrow} a^{2} b^{2} Z_{k}^{-1} Y_{j} Y_{i} \\
& \stackrel{\hat{\alpha}_{j \leftrightarrow k}}{\longrightarrow} a^{2} b^{2} Z_{j}^{-1} Y_{k} Y_{i} \\
& \stackrel{\hat{\varphi}_{j k}}{\longrightarrow} a^{2} b^{2}\left[b\left(b Y_{j} Y_{k}+Z_{j} Z_{k}\right)^{-1} Y_{j}\right]^{-1}\left(b Y_{j} Y_{k}+Z_{j} Z_{k}\right)^{-1} Z_{j} Y_{i}=a^{2} b Y_{j}^{-1} Z_{j} Y_{i} \\
& \stackrel{\hat{\rho}_{j}}{\longrightarrow} a b Z_{j}^{-1} Y_{i} \stackrel{\hat{\alpha}_{i \leftrightarrow j}}{\longrightarrow} a b Z_{i}^{-1} Y_{j} \\
& \stackrel{\hat{\varphi}_{i j}}{\longrightarrow} a b\left[b\left(b Y_{i} Y_{j}+Z_{i} Z_{j}\right)^{-1} Y_{i}\right]^{-1}\left(b Y_{i} Y_{j}+Z_{i} Z_{j}\right)^{-1} Z_{i}=a Y_{i}^{-1} Z_{i} \\
& \stackrel{\hat{\rho}_{i}}{\longrightarrow} Z_{i}^{-1} \stackrel{\hat{\rho}_{i}}{\longrightarrow} Y_{i} .
\end{aligned}
$$

This completes the proof.

Proposition 7 If a decorated ideal triangulation $\tau^{\prime}$ is obtained from another one $\tau$ by an operation $\pi$, where $\pi=\alpha$ for some $\alpha \in \mathfrak{S}_{2 m}$, or $\pi=\rho_{i}$ for some $i$, or $\pi=\varphi_{i j}$ for some $i, j$, then $\hat{\pi}: \widehat{\mathcal{K}}_{\tau^{\prime}}^{q} \rightarrow \widehat{\mathcal{K}}_{\tau}^{q}$ as in Definition 5 is an isomorphism between the two algebras.

Proof If $\pi=\alpha$ for some $\alpha \in \mathfrak{S}_{2 m}$, it is obvious that $\hat{\pi}$ is an isomorphism. 
If $\pi=\rho_{i}$ for some $i$, we need to check that $\hat{\pi}$ is a homomorphism, that is, it preserve the algebraic relations (1). The first three are obvious. It is enough to check the last one. Since $Z_{i}^{\prime} Y_{i}^{\prime}=q^{2} Y_{i}^{\prime} Z_{i}^{\prime}$, we need to show that $\hat{\pi}\left(Z_{i}^{\prime} Y_{i}^{\prime}\right)=q^{2} \hat{\pi}\left(Y_{i}^{\prime} Z_{i}^{\prime}\right)$. We verify this by showing

$$
\begin{array}{rlrl}
\hat{\pi}\left(Z_{i}^{\prime} Y_{i}^{\prime}\right) & =q^{2} \hat{\pi}\left(Y_{i}^{\prime} Z_{i}^{\prime}\right) \\
& \Longleftrightarrow & & \\
& \Longleftrightarrow & \hat{\pi}\left(Z_{i}^{\prime}\right) \hat{\pi}\left(Y_{i}^{\prime}\right) & =q^{2} \hat{\pi}\left(Y_{i}^{\prime}\right) \hat{\pi}\left(Z_{i}^{\prime}\right) \\
& \Longleftrightarrow & Y_{i}^{-1} a Y_{i}^{-1} Z_{i} & =q^{2} a Y_{i}^{-1} Z_{i} Y_{i}^{-1} \quad \text { by Definition 5 } \\
& \Longleftrightarrow & Y_{i}^{-1} Z_{i} & =q^{2} Z_{i} Y_{i}^{-1} \\
Z_{i} Y_{i} & =q^{2} Y_{i} Z_{i} .
\end{array}
$$

This is true.

To show that $\hat{\rho}_{i}$ is an isomorphism, it is enough to find its inverse. In fact, by Proposition 6(6), we see $\hat{\rho}_{i}^{-1}=\hat{\rho}_{i} \circ \widehat{\rho}_{i}$.

If $\pi=\varphi_{i j}$ for some $i, j$, we need to check that $\hat{\pi}$ is a homomorphism, that is, it preserve the algebraic relations (1).

Case 1 For $\{k, l\} \neq\{i, j\}$, since $\hat{\pi}\left(Y_{k}^{\prime}, Z_{k}^{\prime}, Y_{l}^{\prime}, Z_{l}^{\prime}\right)=\left(Y_{k}, Z_{k}, Y_{l}, Z_{l}\right)$, therefore $\hat{\pi}$ preserves the relation of $Y_{k}^{\prime}, Z_{k}^{\prime}, Y_{l}^{\prime}, Z_{l}^{\prime}$.

Case 2 For $k \notin\{i, j\}$, we consider $Y_{k}^{\prime}, Z_{k}^{\prime}$ and $Y_{i}^{\prime}, Z_{i}^{\prime}$. Now

$$
\begin{array}{ll}
\hat{\varphi}_{i j}\left(Y_{k}^{\prime}\right)=Y_{k}, & \hat{\varphi}_{i j}\left(Z_{k}^{\prime}\right)=Z_{k}, \\
\hat{\varphi}_{i j}\left(Y_{i}^{\prime}\right)=\left(b Y_{i} Y_{j}+Z_{i} Z_{j}\right)^{-1} Z_{j}, & \hat{\varphi}_{i j}\left(Z_{i}^{\prime}\right)=b\left(b Y_{i} Y_{j}+Z_{i} Z_{j}\right)^{-1} Y_{i} .
\end{array}
$$

Since $Y_{k}^{\prime} Y_{i}^{\prime}=Y_{i}^{\prime} Y_{k}^{\prime}$, we need to check that $\widehat{\pi}\left(Y_{k}^{\prime} Y_{i}^{\prime}\right)=\widehat{\pi}\left(Y_{i}^{\prime} Y_{k}^{\prime}\right)$. This is true.

Since $Z_{k}^{\prime} Z_{i}^{\prime}=Z_{i}^{\prime} Z_{k}^{\prime}$, we need to check that $\hat{\pi}\left(Z_{k}^{\prime} Z_{i}^{\prime}\right)=\hat{\pi}\left(Z_{i}^{\prime} Z_{k}^{\prime}\right)$. This is true.

Since $Z_{k}^{\prime} Y_{k}^{\prime}=q^{2} Y_{k}^{\prime} Z_{k}^{\prime}$, we need to check that $\hat{\pi}\left(Z_{k}^{\prime} Y_{k}^{\prime}\right)=q^{2} \hat{\pi}\left(Y_{k}^{\prime} Z_{k}^{\prime}\right)$. This is true. Since $Z_{i}^{\prime} Y_{i}^{\prime}=q^{2} Y_{i}^{\prime} Z_{i}^{\prime}$, we need to check that $\hat{\pi}\left(Z_{i}^{\prime} Y_{i}^{\prime}\right)=q^{2} \hat{\pi}\left(Y_{i}^{\prime} Z_{i}^{\prime}\right)$ which is equivalent to

$$
\begin{array}{cc} 
& b\left(b Y_{i} Y_{j}+Z_{i} Z_{j}\right)^{-1} Y_{i}\left(b Y_{i} Y_{j}+Z_{i} Z_{j}\right)^{-1} Z_{j} \\
=q^{2}\left(b Y_{i} Y_{j}+Z_{i} Z_{j}\right)^{-1} Z_{j} b\left(b Y_{i} Y_{j}+Z_{i} Z_{j}\right)^{-1} Y_{i} \\
& \Longleftrightarrow \quad Y_{i}\left(b Y_{i} Y_{j}+Z_{i} Z_{j}\right)^{-1} Z_{j}=q^{2} Z_{j}\left(b Y_{i} Y_{j}+Z_{i} Z_{j}\right)^{-1} Y_{i} \\
\Longleftrightarrow \quad Z_{j} Y_{i}^{-1}\left(b Y_{i} Y_{j}+Z_{i} Z_{j}\right)=q^{2}\left(b Y_{i} Y_{j}+Z_{i} Z_{j}\right) Y_{i}^{-1} Z_{j} .
\end{array}
$$


This is true since

$$
\begin{aligned}
\text { the left hand side } & =Z_{j}\left(b Y_{i} Y_{j}+q^{2} Z_{i} Z_{j}\right) Y_{i}^{-1} \\
& =\left(b q^{2} Y_{i} Y_{j}+q^{2} Z_{i} Z_{j}\right) Z_{j} Y_{i}^{-1} \\
& =q^{2}\left(b Y_{i} Y_{j}+Z_{i} Z_{j}\right) Z_{j} Y_{i}^{-1} \\
& =\text { the right hand side. }
\end{aligned}
$$

Case 3 We consider $Y_{i}^{\prime}, Z_{i}^{\prime}$ and $Y_{j}^{\prime}, Z_{j}^{\prime}$.

Since $Y_{i}^{\prime} Y_{j}^{\prime}=Y_{j}^{\prime} Y_{i}^{\prime}$, we need to check that $\hat{\pi}\left(Y_{i}^{\prime} Y_{j}^{\prime}\right)=\widehat{\pi}\left(Y_{j}^{\prime} Y_{i}^{\prime}\right)$ which is equivalent to

$$
\begin{gathered}
\left(b Y_{i} Y_{j}+Z_{i} Z_{j}\right)^{-1} Z_{j}\left(b Y_{i} Y_{j}+Z_{i} Z_{j}\right)^{-1} Z_{i} \\
=\left(b Y_{i} Y_{j}+Z_{i} Z_{j}\right)^{-1} Z_{j}\left(b Y_{i} Y_{j}+Z_{i} Z_{j}\right)^{-1} Y_{i} \\
\Longleftrightarrow \quad Z_{j}\left(b Y_{i} Y_{j}+Z_{i} Z_{j}\right)^{-1} Z_{i}=Z_{i}\left(b Y_{i} Y_{j}+Z_{i} Z_{j}\right)^{-1} Z_{j} \\
\Longleftrightarrow \quad Z_{i} Z_{j}^{-1}\left(b Y_{i} Y_{j}+Z_{i} Z_{j}\right)=\left(b Y_{i} Y_{j}+Z_{i} Z_{j}\right) Z_{j}^{-1} Z_{i}
\end{gathered}
$$

This is true since

$$
\begin{aligned}
\text { the left hand side } & =Z_{i}\left(b q^{-2} Y_{i} Y_{j}+Z_{i} Z_{j}\right) Z_{j}^{-1} \\
& =\left(b q^{-2} q^{2} Y_{i} Y_{j}+Z_{i} Z_{j}\right) Z_{i} Z_{j}^{-1} \\
& =\text { the right hand side. }
\end{aligned}
$$

The similar calculation is used to check that $\hat{\pi}\left(Z_{i}^{\prime} Z_{j}^{\prime}\right)=\hat{\pi}\left(Z_{j}^{\prime} Z_{i}^{\prime}\right)$.

Since $Y_{i}^{\prime} Z_{j}^{\prime}=Z_{j}^{\prime} Y_{i}^{\prime}$, we need to check that $\hat{\pi}\left(Y_{i}^{\prime} Z_{j}^{\prime}\right)=\hat{\pi}\left(Z_{j}^{\prime} Y_{i}^{\prime}\right)$ which is equivalent to

$$
\begin{gathered}
\left(b Y_{i} Y_{j}+Z_{i} Z_{j}\right)^{-1} Z_{j} b\left(b Y_{i} Y_{j}+Z_{i} Z_{j}\right)^{-1} Y_{j} \\
=b\left(b Y_{i} Y_{j}+Z_{i} Z_{j}\right)^{-1} Y_{j}\left(b Y_{i} Y_{j}+Z_{i} Z_{j}\right)^{-1} Z_{j} \\
\Longleftrightarrow \quad Z_{j}\left(b Y_{i} Y_{j}+Z_{i} Z_{j}\right)^{-1} Y_{j}=Y_{j}\left(b Y_{i} Y_{j}+Z_{i} Z_{j}\right)^{-1} Z_{j} \\
\Longleftrightarrow \quad Y_{j} Z_{j}^{-1}\left(b Y_{i} Y_{j}+Z_{i} Z_{j}\right)=\left(b Y_{i} Y_{j}+Z_{i} Z_{j}\right) Z_{j}^{-1} Y_{j}
\end{gathered}
$$

This is true since

$$
\begin{aligned}
\text { the left hand side } & =Y_{j}\left(b q^{-2} Y_{i} Y_{j}+Z_{i} Z_{j}\right) Z_{j}^{-1} \\
& =\left(b q^{-2} Y_{i} Y_{j}+q^{-2} Z_{i} Z_{j}\right) Y_{j} Z_{j}^{-1} \\
& =\left(b q^{-2} Y_{i} Y_{j}+q^{-2} Z_{i} Z_{j}\right) q^{2} Z_{j}^{-1} Y_{j} \\
& =\text { the right hand side. }
\end{aligned}
$$


The similar calculation is used to check that $\hat{\pi}\left(Y_{j}^{\prime} Z_{i}^{\prime}\right)=\hat{\pi}\left(Z_{i}^{\prime} Y_{j}^{\prime}\right)$.

For $Z_{i}^{\prime} Y_{i}^{\prime}=q^{2} Y_{i}^{\prime} Z_{i}^{\prime}$ and $Z_{j}^{\prime} Y_{j}^{\prime}=q^{2} Y_{j}^{\prime} Z_{j}^{\prime}$, we have done in Case 2 .

To show that $\hat{\varphi}_{i j}$ is an isomorphism, it is enough to find its inverse. In fact, by Proposition 6(2), we see $\hat{\varphi}_{i j}^{-1}=\hat{\alpha}_{i \leftrightarrow j} \circ \widehat{\varphi}_{i j}$, where $\alpha_{i \leftrightarrow j}$ denotes the transposition exchanging $i$ and $j$.

Theorem 8 For two arbitrary complex numbers $a, b$, there is a family of algebra isomorphisms

$$
\Psi_{\tau \tau^{\prime}}^{q}(a, b): \widehat{\mathcal{K}}_{\tau^{\prime}}^{q} \rightarrow \widehat{\mathcal{K}}_{\tau}^{q}
$$

defined as $\tau, \tau^{\prime} \in \triangle(S)$ ranges over all pairs of decorated ideal triangulations, such that:

(1) $\Psi_{\tau \tau^{\prime \prime}}^{q}(a, b)=\Psi_{\tau \tau^{\prime}}^{q}(a, b) \circ \Psi_{\tau^{\prime} \tau^{\prime \prime}}^{q}(a, b)$ for every $\tau, \tau^{\prime}, \tau^{\prime \prime} \in \triangle(S)$;

(2) $\Psi_{\tau \tau^{\prime}}^{q}(a, b)$ is the isomorphism of Definition 5 when $\tau^{\prime}$ is obtained from $\tau$ by a reindexing or a mark rotation or a diagonal exchange.

(3) $\Psi_{\tau \tau^{\prime}}^{q}(a, b)$ depends only on $\tau$ and $\tau^{\prime}$.

Proof For a pair $\tau, \tau^{\prime} \in \Delta(S)$, by Theorem 2, there is a sequence $\tau=\tau_{(0)}, \tau_{(1)}, \ldots$, $\tau_{(n)}=\tau^{\prime}$ where each $\tau_{(k+1)}$ is obtained from $\tau_{(k)}$ by a reindexing or a mark rotation or a diagonal exchange. For each $k=0, \ldots, n-1, \Psi_{\tau_{(k)} \tau_{(k+1)}}^{q}(a, b)$ is defined as in Definition 5. Then $\Psi_{\tau \tau^{\prime}}^{q}(a, b)$ can be defined as the composition of the isomorphisms $\Psi_{\tau_{(k)} \tau_{(k+1)}}^{q}(a, b)$.

If $\tau$ and $\tau^{\prime}$ are connected by another sequence $\tau=\bar{\tau}_{(0)}, \bar{\tau}_{(1)}, \ldots, \bar{\tau}_{(\bar{n})}=\tau^{\prime}$. By Theorem 3 , the two sequences are related by some canonical moves. Proposition 6 shows that each move does not change the composition of the isomorphisms $\Psi_{\tau_{(k)} \tau_{(k+1)}}^{q}(a, b)$. Therefore $\Psi_{\tau \tau^{\prime}}^{q}(a, b)$ is independent of the choice of the sequence connecting $\tau$ and $\tau^{\prime}$, that is, $\Psi_{\tau \tau^{\prime}}^{q}(a, b)$ depends only on $\tau$ and $\tau^{\prime}$.

The generalized Kashaev algebra $\widehat{\mathcal{K}}_{S}^{q}(a, b)$ associated to a surface $S$ is defined as the algebra

$$
\hat{\mathcal{K}}_{S}^{q}(a, b)=\left(\bigsqcup_{\tau \in \Delta(S)} \hat{\mathcal{K}}_{\tau}^{q}(a, b)\right) / \sim
$$

where the relation $\sim$ is defined by the property that, for $X \in \widehat{\mathcal{K}}_{\tau}^{q}(a, b)$ and $X^{\prime} \in$ $\widehat{\mathcal{K}}_{\tau^{\prime}}^{q}(a, b)$,

$$
X \sim X^{\prime} \Leftrightarrow X=\Psi_{\tau, \tau^{\prime}}^{q}(a, b)\left(X^{\prime}\right) .
$$




\section{Kashaev coordinates and shear coordinates}

To understand the quantization using shear coordinates and the quantization using Kashaev coordinates, we first need to understand the relationship between these two coordinates.

\subsection{Decorated ideal triangulations}

Given a decorated ideal triangulation $\tau$, by forgetting the mark at each corner, we obtain an ideal triangulation $\lambda$. We call $\lambda$ the underlying ideal triangulation of $\tau$. let $\lambda_{1}, \lambda_{2}, \ldots, \lambda_{3 m}$ be the components of ideal triangulation $\lambda$. Denote by $\tau_{1}, \ldots, \tau_{2 m}$ the ideal triangles.

For the ideal triangulation $\lambda$, we may consider its dual graph. Each ideal triangle $\tau_{\mu}$ corresponds to a vertex $\tau_{\mu}^{*}$ of the dual graph. Denote by $\lambda_{1}^{*}, \lambda_{2}^{*}, \ldots, \lambda_{3 m}^{*}$ the dual edges. If an edge $\lambda_{i}$ bounds one side of the ideal triangles $\tau_{\mu}$ and one side of $\tau_{\nu}$, then the dual edge $\lambda_{i}^{*}$ connects the vertexes $\tau_{\mu}^{*}$ and $\tau_{\nu}^{*}$.

In a decorated ideal triangulation $\tau$, each ideal triangle $\tau_{\mu}$ (embedded or not) has three sides which correspond to the three half-edges incident to the vertex $\tau_{\mu}^{*}$ of the dual graph. The three sides are numerated by $0,1,2$ in the counterclockwise order such that the 0 -side is opposite to the marked corner.

\subsection{Space of Kashaev coordinates}

Let's recall that a Kashaev coordinate associated to a decorated ideal triangulation $\tau$ is a vector $\left(\ln y_{1}, \ln z_{1}, \ldots, \ln y_{2 m}, \ln z_{2 m}\right) \in \mathbb{R}^{4 m}$, where $\ln y_{\mu}$ and $\ln z_{\mu}$ are associated to the ideal triangle $\tau_{\mu}$. Denote by $\mathcal{K}_{\tau}$ the space of Kahsaev coordinates associated to $\tau$. We see that $\mathcal{K}_{\tau}=\mathbb{R}^{4 m}$.

Given a vector $\left(\ln y_{1}, \ln z_{1}, \ldots, \ln y_{2 m}, \ln z_{2 m}\right) \in \mathcal{K}_{\tau}$, we associate a number to each side of each ideal triangle as follows. For the ideal triangle $\tau_{\mu}$, we associate

- $\ln h_{\mu}^{0}:=\ln y_{\mu}-\ln z_{\mu}$ to the $0-$ side;

- $\ln h_{\mu}^{1}:=\ln z_{\mu}$ to the 1 -side;

- $\ln h_{\mu}^{2}:=-\ln y_{\mu}$ to the 2 -side.

Therefore $\ln h_{\mu}^{0}+\ln h_{\mu}^{1}+\ln h_{\mu}^{2}=0$. We can identify the space $\mathcal{K}_{\tau}=\mathbb{R}^{4 m}$ with a subspace of $\mathbb{R}^{6 m}=\left\{\left(\ldots, \ln h_{\mu}^{0}, \ln h_{\mu}^{1}, \ln h_{\mu}^{2}, \ldots\right)\right\}$ satisfying $\ln h_{\mu}^{0}+\ln h_{\mu}^{1}+\ln h_{\mu}^{2}=0$ for each ideal triangle $\tau_{\mu}$. 


\subsection{Exact sequence}

The enhanced Teichmüller space parametrized by shear coordinates is $\widetilde{\mathcal{T}}_{\lambda}=\mathbb{R}^{3 m}=$ $\left\{\left(\ln x_{1}, \ln x_{2}, \ldots, \ln x_{3 m}\right)\right\}$, where $\ln x_{i}$ is the shear coordinate at edge $\lambda_{i}$. We define a map $f_{1}: \widetilde{\mathcal{T}}_{\lambda} \rightarrow \mathbb{R}$ by sending $\left(\ln x_{1}, \ln x_{2}, \ldots, \ln x_{3 m}\right)$ to the sum of entries $\sum_{i=1}^{3 m} \ln x_{i}$.

Suppose $\lambda$ is the underlying ideal triangulation of the decorated ideal triangulation $\tau$. We define a map $f_{2}: \mathcal{K}_{\tau} \rightarrow \widetilde{\mathcal{T}}_{\lambda}$ as a linear function by setting

$$
\ln x_{i}=\ln h_{\mu}^{s}+\ln h_{v}^{t}
$$

whenever $\lambda_{i}$ bounds the $s$-side of $\tau_{\mu}$ and the $t$-side of $\tau_{v}$ ( $\mu$ may equal $v$ ), where $s, t \in\{0,1,2\}$.

Another map $f_{3}: H_{1}(S, \mathbb{R}) \rightarrow \mathcal{K}_{\tau}$ is defined as follows. A homology class in $H_{1}(S, \mathbb{R})$ is represented by a linear combination of oriented dual edges: $\sum_{i=1}^{3 m} c_{i} \lambda_{i}^{*}$. If the orientation of $\lambda_{i}^{*}$ is from the $s$-side of $\tau_{\mu}$ to the $t$-side of $\tau_{\nu}$, by setting $\ln h_{\mu}^{s}=-c_{i}$ and $\ln h_{v}^{t}=c_{i}$, we obtain a vector $\left(\ldots, \ln h_{\mu}^{0}, \ln h_{\mu}^{1}, \ln h_{\mu}^{2}, \ldots\right) \in \mathbb{R}^{6 m}$. The boundary map of chain complexes sends $\sum_{i=1}^{3 m} c_{i} \lambda_{i}^{*}$ to a linear combination of vertexes. In this combination, the term involving the vertex $\tau_{\mu}^{*}$ is $\left(c_{i} \epsilon_{i}+c_{j} \epsilon_{j}+c_{k} \epsilon_{k}\right) \tau_{\mu}^{*}$ where $\lambda_{i}, \lambda_{j}, \lambda_{k}$ (two of them may coincide) bound three sides of $\tau_{\mu}$ and $\epsilon_{t}=-1$ if $\lambda_{t}^{*}$ starts at the side of $\tau_{\mu}$ bounded by $\lambda_{t}$ while $\epsilon_{t}=1$ if $\lambda_{t}^{*}$ ends at the side of $\tau_{\mu}$ bounded by $\lambda_{t}$. Therefore

$$
\left(c_{i} \epsilon_{i}+c_{j} \epsilon_{j}+c_{k} \epsilon_{k}\right) \tau_{\mu}^{*}=\left(\ln h_{\mu}^{0}+\ln h_{\mu}^{1}+\ln h_{\mu}^{2}\right) \tau_{\mu}^{*} .
$$

Since the chain $\sum_{i=1}^{3 m} c_{i} \lambda_{i}^{*}$ is a cycle, we must have $\ln h_{\mu}^{0}+\ln h_{\mu}^{1}+\ln h_{\mu}^{2}=0$. Therefore this vector $\left(\ldots, \ln h_{\mu}^{0}, \ln h_{\mu}^{1}, \ln h_{\mu}^{2}, \ldots\right)$ is in the subspace $\mathcal{K}_{\tau}$.

Combining the three maps, we obtain

Theorem 9 The following sequence is exact:

$$
0 \rightarrow H_{1}(S, \mathbb{R}) \stackrel{f_{3}}{\longrightarrow} \mathcal{K}_{\tau} \stackrel{f_{2}}{\longrightarrow} \widetilde{\mathcal{T}}_{\lambda} \stackrel{f_{1}}{\longrightarrow} \mathbb{R} \rightarrow 0 .
$$

Proof The map $f_{3}$ is injective. In fact, if the homology class represented by $\sum_{i=1}^{3 m} c_{i} \lambda_{i}^{*}$ is mapped to the zero vector in $\mathcal{K}_{\tau}$, then, for each $i=1, \ldots, 3 m$, we have $\left|c_{i}\right|=\left|\ln h_{\mu}^{s}\right|=0$, where $\lambda_{i}$ bounds the $s$-side of $\tau_{\mu}$. Therefore it is a zero homology class. Thus the sequence is exact at $H_{1}(S, \mathbb{R})$. 
Suppose $\left(\ldots, \ln h_{\mu}^{0}, \ln h_{\mu}^{1}, \ln h_{\mu}^{2}, \ldots\right) \in \operatorname{Im}\left(f_{3}\right)$, that is,

$$
\left(\ldots, \ln h_{\mu}^{0}, \ln h_{\mu}^{1}, \ln h_{\mu}^{2}, \ldots\right)=f_{3}\left(\sum_{i=1}^{3 m} c_{i} \lambda_{i}^{*}\right) .
$$

For any edge $\lambda_{i}$ bounds the $s$-side of $\tau_{\mu}$ and the $t$-side of $\tau_{\nu}$, we have

$$
\ln x_{i}=\ln h_{\mu}^{s}+\ln h_{v}^{t}= \pm c_{i} \mp c_{i}=0 .
$$

Thus $\left(\ldots, \ln h_{\mu}^{i}, \ln h_{\mu}^{j}, \ln h_{\mu}^{k}, \ldots\right) \in \operatorname{Ker}\left(f_{2}\right)$. Therefore $\operatorname{Im}\left(f_{3}\right) \subseteq \operatorname{Ker}\left(f_{2}\right)$.

On the other hand, we claim $\operatorname{Im}\left(f_{3}\right) \supseteq \operatorname{Ker}\left(f_{2}\right)$. In fact, given a vector $\left(\ldots, \ln h_{\mu}^{0}\right.$, $\left.\ln h_{\mu}^{1}, \ln h_{\mu}^{2}, \ldots\right) \in \operatorname{Ker}\left(f_{2}\right)$, we can reverse the process of the definition of $f_{3}$ to obtain a homology class in $H_{1}(S, \mathbb{R})$. To be precise, since the vector is in the kernel of $f_{2}$, we have $\ln h_{\mu}^{s}+\ln h_{\nu}^{t}=0$ for each edge $\lambda_{i}$ bounds the $s$-side of $\tau_{\mu}$ and the $t$-side of $\tau_{\nu}$. An orientation of $\lambda_{i}^{*}$ can be given as follows.

- When $\ln h_{\mu}^{s}>0$, the dual edge $\lambda_{i}^{*}$ runs from the $s-$ side of $\tau_{\mu}$ to the $t-$ side of $\tau_{v}$.

- When $\ln h_{\mu}^{s}<0$, the dual edge $\lambda_{i}^{*}$ runs from the $t$-side of $\tau_{\nu}$ to the $s-$ side of $\tau_{\mu}$.

- When $\ln h_{\mu}^{s}=0, \lambda_{i}^{*}$ is oriented in either way.

Consider the one dimensional chain $\sum_{i=1}^{3 m}\left|\ln h_{\mu}^{s}\right| \lambda_{i}^{*}$, where $\lambda_{i}$ bounds the $s$-side of $\tau_{\mu}$. This chain turns out to be a cycle. In fact, the boundary map sends this chain to a zero dimensional chain in which the term involving the vertex $\tau_{\mu}^{*}$ is

$$
\left(\left|\ln h_{\mu}^{0}\right| \epsilon_{0}+\left|\ln h_{\mu}^{1}\right| \epsilon_{1}+\left|\ln h_{\mu}^{2}\right| \epsilon_{2}\right) \tau_{\mu}^{*}
$$

where $\epsilon_{s}= \pm 1$ and $\epsilon_{s}=\operatorname{sig} n\left(\ln h_{\mu}^{s}\right) \cdot 1$ if $\ln h_{\mu}^{s} \neq 0$ for $s \in\{0,1,2\}$. Thus

$$
\left(\left|\ln h_{\mu}^{0}\right| \epsilon_{0}+\left|\ln h_{\mu}^{1}\right| \epsilon_{1}+\left|\ln h_{\mu}^{2}\right| \epsilon_{2}\right) \tau_{\mu}^{*}=\left(\ln h_{\mu}^{0}+\ln h_{\mu}^{1}+\ln h_{\mu}^{2}\right) \tau_{\mu}^{*}=0 .
$$

This cycle defines a homology class.

The argument above shows $\operatorname{Im}\left(f_{3}\right)=\operatorname{Ker}\left(f_{2}\right)$, that is, the sequence is exact at $\mathcal{K}_{\tau}$.

Now $\operatorname{dim} \operatorname{Ker}\left(f_{2}\right)=\operatorname{dim} \operatorname{Im}\left(f_{3}\right)=\operatorname{dim} H_{1}(S, \mathbb{R})=2 g+p-1=m+1$. Thus $\operatorname{dim} \operatorname{Im}\left(f_{2}\right)=\operatorname{dim} \mathcal{K}_{\tau}-\operatorname{dim} \operatorname{Ker}\left(f_{2}\right)=4 m-(m+1)=3 m-1$. Since

$$
\operatorname{Ker}\left(f_{1}\right)=\left\{\left(x_{1}, x_{2}, \ldots, x_{3 m}\right) \mid \sum_{i=1}^{3 m} x_{i}=0\right\}
$$


is a subspace of dimension $3 m-1$, then $\operatorname{Im}\left(f_{2}\right)=\operatorname{Ker}\left(f_{1}\right)$, that is, the sequence is exact at $\widetilde{\mathcal{T}_{\lambda}}$.

It is easy to see that $f_{1}$ is onto. Therefore the sequence is exact at $\mathbb{R}$.

Remark From the theorem above, we see that $\mathcal{K}_{\tau}$ is a fiber bundle over the space $\operatorname{Ker}\left(f_{1}\right)$ whose fiber is an affine space modeled on $H_{1}(S, \mathbb{R})$. To be precise, given a vector $s \in \operatorname{Ker}\left(f_{1}\right)$, let $v \in f_{2}^{-1}(s)$. Then $f_{2}^{-1}(s)=v+H_{1}(S, \mathbb{R})$.

Remark There is an exact sequence relating space of Kashaev coordinates and decorated Teichmüller space. See Proposition 21 in the Appendix of this paper.

\subsection{Relation of bivecotrs}

Consider the linear isomorphism

$$
M: \mathcal{K}_{\tau} \longrightarrow \mathcal{K}_{\tau}
$$

$$
\left(\ln y_{1}, \ln z_{1}, \ldots, \ln y_{2 m}, \ln z_{2 m}\right) \longmapsto\left(\ldots, \ln h_{\mu}^{0}, \ln h_{\mu}^{1}, \ln h_{\mu}^{2}, \ldots\right) .
$$

\section{Proposition 10 If}

$$
\left(\ln x_{1}, \ln x_{2}, \ldots, \ln x_{3 m}\right)=f_{2} \circ M\left(\ln y_{1}, \ln z_{1}, \ldots, \ln y_{2 m}, \ln z_{2 m}\right),
$$

then

$$
\sum_{i, j=1}^{3 m} \sigma_{i j}^{\lambda} \frac{\partial}{\partial \ln x_{i}} \wedge \frac{\partial}{\partial \ln x_{j}}=\left(f_{2}\right)_{*} \circ M_{*}\left(\sum_{\mu=1}^{2 m} \frac{\partial}{\partial \ln y_{\mu}} \wedge \frac{\partial}{\partial \ln z_{\mu}}\right),
$$

where $\sigma_{i j}^{\lambda}=a_{i j}^{\lambda}-a_{j i}^{\lambda}$ and $a_{i j}^{\lambda}$ is the number of corners of the ideal triangulation $\lambda$ which is delimited in the left by $\lambda_{i}$ and on the right by $\lambda_{j}$.

Proof By definition (2), we have

$M_{*}\left(\frac{\partial}{\partial \ln y_{\mu}} \wedge \frac{\partial}{\partial \ln z_{\mu}}\right)=\frac{\partial}{\partial \ln h_{\mu}^{0}} \wedge \frac{\partial}{\partial \ln h_{\mu}^{1}}+\frac{\partial}{\partial \ln h_{\mu}^{1}} \wedge \frac{\partial}{\partial \ln h_{\mu}^{2}}+\frac{\partial}{\partial \ln h_{\mu}^{2}} \wedge \frac{\partial}{\partial \ln h_{\mu}^{1}}$. Assume that the edges $\lambda_{i}, \lambda_{j}, \lambda_{k}$ (two of them may coincide) bound the 0 -side, 1 -side and 2 -side of the ideal triangle $\tau_{\mu}$ respectively.

By definition of map $f_{2}$, we have

$$
\begin{array}{r}
\left(f_{2}\right)_{*}\left(\frac{\partial}{\partial \ln h_{\mu}^{0}} \wedge \frac{\partial}{\partial \ln h_{\mu}^{1}}+\frac{\partial}{\partial \ln h_{\mu}^{1}} \wedge \frac{\partial}{\partial \ln h_{\mu}^{2}}+\frac{\partial}{\partial \ln h_{\mu}^{2}} \wedge \frac{\partial}{\partial \ln h_{\mu}^{0}}\right) \\
=\frac{\partial}{\partial \ln x_{i}} \wedge \frac{\partial}{\partial \ln x_{j}}+\frac{\partial}{\partial \ln x_{j}} \wedge \frac{\partial}{\partial \ln x_{k}}+\frac{\partial}{\partial \ln x_{k}} \wedge \frac{\partial}{\partial \ln x_{i}} .
\end{array}
$$


Therefore

$$
\begin{aligned}
& \left(f_{2}\right)_{*} \circ M_{*}\left(\sum_{\mu=1}^{2 m} \frac{\partial}{\partial \ln y_{\mu}} \wedge \frac{\partial}{\partial \ln z_{\mu}}\right) \\
= & \sum_{\mu=1}^{2 m}\left(\frac{\partial}{\partial \ln x_{i}} \wedge \frac{\partial}{\partial \ln x_{j}}+\frac{\partial}{\partial \ln x_{j}} \wedge \frac{\partial}{\partial \ln x_{k}}+\frac{\partial}{\partial \ln x_{k}} \wedge \frac{\partial}{\partial \ln x_{i}}\right) \\
& \text { where } \lambda_{i}, \lambda_{j}, \lambda_{k} \text { bound the 0-side, 1-side and 2-side of } \tau_{\mu} \\
= & \sum_{i, j=1}^{3 m} \sigma_{i j}^{\lambda} \frac{\partial}{\partial \ln x_{i}} \wedge \frac{\partial}{\partial \ln x_{j}} .
\end{aligned}
$$

This completes the proof.

Remark There is a relationship between a differential 2-form in Kashaev coordinates and the Weil-Petersson 2-form in Penner coordinates. See Proposition 22 in the Appendix of this paper.

\subsection{Compatibility of coordinate changes}
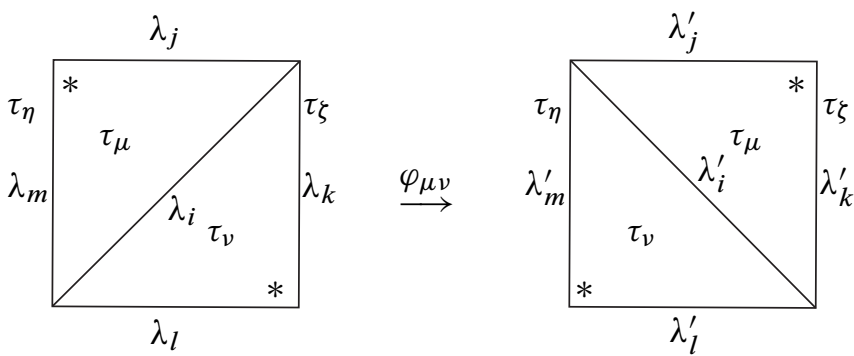

Figure 4

The coordinate change of shear coordinates are given as

Proposition 11 (Liu [14, Proposition 3]) Suppose that the ideal triangulations $\lambda$, $\lambda^{\prime}$ are obtained from each other by a diagonal exchange, namely that $\lambda^{\prime}=\Delta_{i}(\lambda)$. Label the edges of $\lambda$ involved in this diagonal exchange as $\lambda_{i}, \lambda_{j}, \lambda_{k}, \lambda_{l}, \lambda_{m}$ as in Figure 4. If $\left(x_{1}, x_{2}, \ldots, x_{n}\right)$ and $\left(x_{1}^{\prime}, x_{2}^{\prime}, \ldots, x_{n}^{\prime}\right)$ are the exponential shear coordinates associated $\lambda$ and $\lambda^{\prime}$ of the same enhanced hyperbolic metric, then $x_{h}^{\prime}=x_{h}$ for every $h \notin\{i, j, k, l, m\}, x_{i}^{\prime}=x_{i}^{-1}$ and:

(1) if the edges $\lambda_{j}, \lambda_{k}, \lambda_{l}, \lambda_{m}$ are distinct, then $x_{j}^{\prime}=\left(1+x_{i}\right) x_{j} \quad x_{k}^{\prime}=\left(1+x_{i}^{-1}\right)^{-1} x_{k} \quad x_{l}^{\prime}=\left(1+x_{i}\right) x_{l} \quad x_{m}^{\prime}=\left(1+x_{i}^{-1}\right)^{-1} x_{m} ;$ 
(2) if $\lambda_{j}$ is identified with $\lambda_{k}$, and $\lambda_{l}$ is distinct from $\lambda_{m}$, then

$$
x_{j}^{\prime}=x_{i} x_{j} \quad x_{l}^{\prime}=\left(1+x_{i}\right) x_{l} \quad x_{m}^{\prime}=\left(1+x_{i}^{-1}\right)^{-1} x_{m} ;
$$

(3) (the inverse of (2)) if $\lambda_{j}$ is identified with $\lambda_{m}$, and $\lambda_{k}$ is distinct from $\lambda_{l}$, then

$$
x_{j}^{\prime}=x_{i} x_{j} \quad x_{k}^{\prime}=\left(1+x_{i}^{-1}\right)^{-1} x_{k} \quad x_{l}^{\prime}=\left(1+x_{i}\right) x_{l} ;
$$

(4) if $\lambda_{j}$ is identified with $\lambda_{l}$, and $\lambda_{k}$ is distinct from $\lambda_{m}$, then

$$
x_{j}^{\prime}=\left(1+x_{i}\right)^{2} x_{j} \quad x_{k}^{\prime}=\left(1+x_{i}^{-1}\right)^{-1} x_{k} \quad x_{m}^{\prime}=\left(1+x_{i}^{-1}\right)^{-1} x_{m}
$$

(5) (the inverse of (4)) if $\lambda_{k}$ is identified with $\lambda_{m}$, and $\lambda_{j}$ is distinct from $\lambda_{l}$, then

$$
x_{j}^{\prime}=\left(1+x_{i}\right) x_{j} \quad x_{k}^{\prime}=\left(1+x_{i}^{-1}\right)^{-2} x_{k} \quad x_{l}^{\prime}=\left(1+x_{i}\right) x_{l} ;
$$

(6) if $\lambda_{j}$ is identified with $\lambda_{k}$, and $\lambda_{l}$ is identified with $\lambda_{m}$ (in which case $S$ is a 3-times punctured sphere), then

$$
x_{j}^{\prime}=x_{i} x_{j} \quad x_{l}^{\prime}=x_{i} x_{l}
$$

(7) (the inverse of (6)) if $\lambda_{j}$ is identified with $\lambda_{m}$, and $\lambda_{k}$ is identified with $\lambda_{l}$ (in which case $S$ is a 3-times punctured sphere), then

$$
x_{j}^{\prime}=x_{i} x_{j} \quad x_{k}^{\prime}=x_{i} x_{k}
$$

(8) if $\lambda_{j}$ is identified with $\lambda_{l}$, and $\lambda_{k}$ is identified with $\lambda_{m}$ (in which case $S$ is a once punctured torus), then

$$
x_{j}^{\prime}=\left(1+x_{i}\right)^{2} x_{j} \quad x_{k}^{\prime}=\left(1+x_{i}^{-1}\right)^{-2} x_{k} .
$$

Proposition 12 Suppose that the decorated ideal triangulations $\tau$ and $\tau^{\prime}$ have the underlying ideal triangulations $\lambda$ and $\lambda^{\prime}$ respectively. The following diagram is commutative:

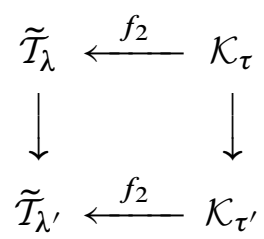

where the two vertical maps are corresponding coordinate changes. The coordinate changes of Kashaev coordinates are given in Definition 4. The coordinate changes of shear coordinates are given in Proposition 11.

Proof For a reindexing, the conclusion is obvious. For a mark rotation, the conclusion is easily proved by definition. For diagonal exchange, we need to check the eight cases 
in Proposition 11. For instance, we verify Case 4. As in Figure 4, through maps $f_{2}$ and $M$, we may identify

$$
\begin{aligned}
& x_{i}=\frac{y_{\mu} y_{v}}{z_{\mu} z_{v}} \quad x_{i}^{\prime}=\frac{y_{\mu}^{\prime} y_{v}^{\prime}}{z_{\mu}^{\prime} z_{v}^{\prime}} \quad x_{j}=z_{\mu} z_{v} \quad x_{j}^{\prime}=\frac{1}{y_{\mu}^{\prime} y_{v}^{\prime}} \\
& x_{k}=\frac{h_{\zeta}^{s}}{y_{\nu}} \quad x_{k}^{\prime}=z_{\mu}^{\prime} h_{\zeta}^{s} \quad x_{m}=\frac{h_{\eta}^{t}}{y_{\mu}} \quad x_{m}^{\prime}=z_{v}^{\prime} h_{\eta}^{t}
\end{aligned}
$$

for some $s, t \in\{0,1,2\}$.

Then we have

$$
\varphi_{\mu \nu}\left(x_{i}^{\prime}\right)=\varphi_{\mu \nu}\left(\frac{y_{\mu}^{\prime} y_{v}^{\prime}}{z_{\mu}^{\prime} z_{v}^{\prime}}\right)=\frac{z_{\mu} z_{v}}{y_{\mu} y_{v}}=x_{i}^{-1}
$$

And

$$
\varphi_{\mu \nu}\left(x_{j}^{\prime}\right)=\varphi_{\mu \nu}\left(\frac{1}{y_{\mu}^{\prime} y_{v}^{\prime}}\right)=\frac{\left(y_{\mu} y_{\nu}+z_{\mu} z_{v}\right)^{2}}{z_{\mu} z_{v}}=\left(1+\frac{y_{\mu} y_{v}}{z_{\mu} z_{v}}\right)^{2} z_{\mu} z_{v}=\left(1+x_{i}\right)^{2} x_{j} \text {. }
$$

And

$$
\varphi_{\mu \nu}\left(x_{k}^{\prime}\right)=\varphi_{\mu \nu}\left(z_{\mu}^{\prime}\right) h_{\zeta}^{s}=\frac{y_{\mu} y_{\nu}}{y_{\mu} y_{\nu}+z_{\mu} z_{\nu}} \frac{1}{y_{\mu}} h_{\zeta}^{s}=\left(1+x_{i}^{-1}\right)^{-1} x_{k}
$$

It is same for $x_{m}^{\prime}$ due to the symmetry of $\mu, v$.

Remark The compatibility of coordinate changes of Kashaev coordinates and Penner coordinates is given in Proposition 23 in Appendix A.

\section{The relationship between quantum Teichmüller space and Kashaev algebra}

In this section, we establish a natural relationship between the quantum Teichmüller space $\hat{\mathcal{T}}_{S}^{q}$ and the generalized Kashaev algebra $\hat{\mathcal{K}}_{S}^{q}(a, b)$.

\subsection{Homomorphism}

For a decorated ideal triangulation $\tau$ of a punctured surface $S$, Kashaev [9] introduced an algebra $\mathcal{K}_{\tau}^{q}$ on $\mathbb{C}$ generated by $Y_{1}^{ \pm}, Z_{1}^{ \pm}, Y_{2}^{ \pm}, Z_{2}^{ \pm}, \ldots, Y_{2 m}^{ \pm}, Z_{2 m}^{ \pm}$, with $Y_{i}^{ \pm}, Z_{i}^{ \pm}$ associated to an ideal triangle $\tau_{i}$, subject to the relations (1).

For a ideal triangle $\tau_{\mu}$, we associate three elements in $\mathcal{K}_{\tau}^{q}$ to the three sides of $\tau_{\mu}$ as follows: 
- $H_{\mu}^{0}:=Y_{\mu} Z_{\mu}^{-1}$ to the 0 -side;

- $H_{\mu}^{1}:=Z_{\mu}$ to the 1 -side;

- $H_{\mu}^{2}:=Y_{\mu}^{-1}$ to the 2 -side.

Lemma 13 For any $s, t \in\{0,1,2\}$ and $\mu \in 1,2, \ldots, 3 m$,

$$
H_{\mu}^{s} H_{\mu}^{t}=q^{2 \sigma_{s t}} H_{\mu}^{t} H_{\mu}^{s},
$$

where $\sigma_{s t}+\sigma_{t s}=0$ and $\sigma_{10}=\sigma_{02}=\sigma_{21}=1$.

Proof When $(s, t)=(1,0)$, we have $H_{\mu}^{s}=Z_{\mu}$ and $H_{\mu}^{t}=Y_{\mu} Z_{\mu}^{-1}$. Thus

$$
H_{\mu}^{s} H_{\mu}^{t}=Z_{\mu} Y_{\mu} Z_{\mu}^{-1}=q^{2} Y_{\mu} Z_{\mu}^{-1} Z_{\mu}=q^{2} H_{\mu}^{t} H_{\mu}^{s} .
$$

When $(s, t)=(0,2)$, we have $H_{\mu}^{s}=Y_{\mu} Z_{\mu}^{-1}$ and $H_{\mu}^{t}=Y_{\mu}^{-1}$. Thus

$$
H_{\mu}^{s} H_{\mu}^{t}=Y_{\mu} Z_{\mu}^{-1} Y_{\mu}^{-1}=q^{2} Y_{\mu}^{-1} Y_{\mu} Z_{\mu}^{-1}=q^{2} H_{\mu}^{t} H_{\mu}^{s} .
$$

When $(s, t)=(2,1)$, we have $H_{\mu}^{s}=Y_{\mu}^{-1}$ and $H_{\mu}^{t}=Z_{\mu}$. Thus

$$
H_{\mu}^{s} H_{\mu}^{t}=Y_{\mu}^{-1} Z_{\mu}=q^{2} Z_{\mu} Y_{\mu}^{-1}=q^{2} H_{\mu}^{t} H_{\mu}^{s} .
$$

This completes the proof.

Suppose $\lambda$ is the underlying ideal triangulation of $\tau$, the Chekhov-Fock algebra $\mathcal{T}_{\lambda}^{q}$ is the algebra over $\mathbb{C}$ defined by generators $X_{1}^{ \pm 1}, X_{2}^{ \pm 1}, \ldots, X_{n}^{ \pm 1}$ associated to the components of $\lambda$ and by relations $X_{i} X_{j}=q^{2 \sigma_{i j}^{\lambda}} X_{j} X_{i}$.

We define a map $F_{\tau}: \mathcal{T}_{\lambda}^{q} \rightarrow \mathcal{K}_{\tau}^{q}$ by indicating the image of the generators and extend it to the whole algebra. Suppose that the edge $\lambda_{i}$ bounds the $s-$ side of $\tau_{\mu}$ and the $t$-side of $\tau_{v}$. We define

$$
F_{\tau}\left(X_{i}\right)=q^{\delta_{\mu \nu} \sigma_{t s}} H_{\mu}^{s} H_{\nu}^{t} \in \mathcal{K}_{\tau}^{q},
$$

where $\sigma_{t s}$ is defined in Lemma 13 and $\delta_{\mu \nu}$ is the Kronecker delta, that is, $\delta_{\mu \mu}=1$ and $\delta_{\mu \nu}=0$ if $\mu \neq v$. When $\mu=v, X_{i}$ is well-defined, since

$$
q^{\sigma_{t s}} H_{\mu}^{s} H_{\mu}^{t}=q^{\sigma_{s t}} H_{\mu}^{t} H_{\mu}^{s}
$$

due to Lemma 13.

This definition is natural since when $q=1$ we get the relationship between Kashaev coordinates and shear coordinates which is given by the map $M$ and $f_{2}$. In fact when $q=1$ then generators $Y_{\mu}, Z_{\mu}$ are commutative. They reduce to the geometric quantities $y_{\mu}, z_{\mu}$ associate to $\tau_{\mu} . H_{\mu}^{s}$ and $X_{i}$ are reduced to $h_{\mu}^{s}$ and $x_{i}$. 
Lemma 14 The map $F_{\tau}: \mathcal{T}_{\lambda}^{q} \rightarrow \mathcal{K}_{\tau}^{q}$ is a homomorphism.

Proof It is enough to check $F_{\tau}\left(X_{i}\right) F_{\tau}\left(X_{j}\right)=q^{2 \sigma_{i j}^{\lambda}} F_{\tau}\left(X_{j}\right) F_{\tau}\left(X_{i}\right)$ for any elements $X_{i}$ and $X_{j}$. Assume the edge $\lambda_{i}$ bounds the $s$-side of $\tau_{\mu}$ and the $t$-side of $\tau_{\nu}$ while the edge $\lambda_{j}$ bounds the $k$-side of $\tau_{\zeta}$ and the $l$-side of $\tau_{\eta}$.

If $\{\mu, \nu\} \cap\{\zeta, \eta\}=\varnothing$, then $F_{\tau}\left(X_{i}\right)$ commutes with $F_{\tau}\left(X_{j}\right)$. On the other hand, $\sigma_{i j}^{\lambda}=0$. The statement holds.

If $(\mu, v, \zeta, \eta)=(\mu, \mu, \mu, \mu)$, then $X_{i}=X_{j}$. The statement holds.

If $(\mu, \nu, \zeta, \eta)=(\mu, \mu, \mu, \eta), \mu \neq \eta$, then $F_{\tau}\left(X_{i}\right)=q^{\sigma_{t s}} H_{\mu}^{s} H_{\mu}^{t}$ and $F_{\tau}\left(X_{j}\right)=H_{\mu}^{k} H_{\eta}^{l}$. Thus by Lemma 13, we have

$$
\begin{aligned}
F_{\tau}\left(X_{i}\right) F_{\tau}\left(X_{j}\right) & =q^{2\left(\sigma_{t k}+\sigma_{s k}\right)} F_{\tau}\left(X_{j}\right) F_{\tau}\left(X_{i}\right) \\
& =F_{\tau}\left(X_{j}\right) F_{\tau}\left(X_{i}\right)=q^{2 \sigma_{i j}^{\lambda}} F_{\tau}\left(X_{j}\right) F_{\tau}\left(X_{i}\right),
\end{aligned}
$$

due to $\sigma_{i j}^{\lambda}=0$.

If $(\mu, \nu, \zeta, \eta)=(\mu, v, v, \eta), \mu \neq v, \mu \neq \eta, v \neq \eta$, then $F_{\tau}\left(X_{i}\right)=H_{\mu}^{s} H_{v}^{t}$ and $F_{\tau}\left(X_{j}\right)=$ $H_{v}^{k} H_{\eta}^{l}$. Thus by Lemma 13 , we have

$$
F_{\tau}\left(X_{i}\right) F_{\tau}\left(X_{j}\right)=q^{2 \sigma_{t k}} F_{\tau}\left(X_{j}\right) F_{\tau}\left(X_{i}\right)=q^{2 \sigma_{i j}^{\lambda}} F_{\tau}\left(X_{j}\right) F_{\tau}\left(X_{i}\right) .
$$

If $(\mu, v, \zeta, \eta)=(\mu, v, \mu, v), \mu \neq v$, then $F_{\tau}\left(X_{i}\right)=H_{\mu}^{s} H_{v}^{t}$ and $F_{\tau}\left(X_{j}\right)=H_{\mu}^{k} H_{v}^{l}$. Thus by Lemma 13, we have

$$
F_{\tau}\left(X_{i}\right) F_{\tau}\left(X_{j}\right)=q^{2\left(\sigma_{s k}+\sigma_{t l}\right)} F_{\tau}\left(X_{j}\right) F_{\tau}\left(X_{i}\right)=q^{2 \sigma_{i j}^{\lambda}} F_{\tau}\left(X_{j}\right) F_{\tau}\left(X_{i}\right) .
$$

\subsection{Compatibility}

Chekhov-Fock algebra $\mathcal{T}_{\lambda}^{q}$ has a well-defined fraction division algebra $\hat{\mathcal{T}}_{\lambda}^{q}$. As one moves from one ideal triangulation $\lambda$ to another $\lambda^{\prime}$, Chekhov and Fock $[7 ; 8 ; 6]$ (see also [14]) introduce coordinate change isomorphisms $\Phi_{\lambda \lambda^{\prime}}^{q}: \widehat{\mathcal{T}}_{\lambda^{\prime}}^{q} \rightarrow \widehat{\mathcal{T}}_{\lambda}^{q}$.

Proposition 15 (Liu [14, Proposition 5]) Suppose that the ideal triangulations $\lambda, \lambda^{\prime}$ are obtained from each other by a diagonal exchange, namely that $\lambda^{\prime}=\Delta_{i}(\lambda)$. Label the edges of $\lambda$ involved in this diagonal exchange as $\lambda_{i}, \lambda_{j}, \lambda_{k}, \lambda_{l}, \lambda_{m}$ as in Figure 4. Then there is a unique algebra isomorphism

$$
\widehat{\Delta}_{i}: \hat{\mathcal{T}}_{\lambda^{\prime}}^{q} \rightarrow \hat{\mathcal{T}}_{\lambda}^{q}
$$

such that $X_{h}^{\prime} \mapsto X_{h}$ for every $h \notin\{i, j, k, l, m\}, X_{i}^{\prime} \mapsto X_{i}^{-1}$ and: 
(1) if the edges $\lambda_{j}, \lambda_{k}, \lambda_{l}, \lambda_{m}$ are distinct, then

$$
\begin{aligned}
X_{j}^{\prime} & \mapsto\left(1+q X_{i}\right) X_{j} & X_{k}^{\prime} & \mapsto\left(1+q X_{i}^{-1}\right)^{-1} X_{k} \\
X_{l}^{\prime} & \mapsto\left(1+q X_{i}\right) X_{l} & X_{m}^{\prime} & \mapsto\left(1+q X_{i}^{-1}\right)^{-1} X_{m} ;
\end{aligned}
$$

(2) if $\lambda_{j}$ is identified with $\lambda_{k}$, and $\lambda_{l}$ is distinct from $\lambda_{m}$, then

$$
X_{j}^{\prime} \mapsto X_{i} X_{j} \quad X_{l}^{\prime} \mapsto\left(1+q X_{i}\right) X_{l} \quad X_{m}^{\prime} \mapsto\left(1+q X_{i}^{-1}\right)^{-1} X_{m}
$$

(3) (the inverse of (2)) if $\lambda_{j}$ is identified with $\lambda_{m}$, and $\lambda_{k}$ is distinct from $\lambda_{l}$, then

$$
X_{j}^{\prime} \mapsto X_{i} X_{j} \quad X_{k}^{\prime} \mapsto\left(1+q X_{i}^{-1}\right)^{-1} X_{k} \quad X_{l}^{\prime} \mapsto\left(1+q X_{i}\right) X_{l}
$$

(4) if $\lambda_{j}$ is identified with $\lambda_{l}$, and $\lambda_{k}$ is distinct from $\lambda_{m}$, then

$$
\begin{gathered}
X_{j}^{\prime} \mapsto\left(1+q X_{i}\right)\left(1+q^{3} X_{i}\right) X_{j} \\
X_{k}^{\prime} \mapsto\left(1+q X_{i}^{-1}\right)^{-1} X_{k} \quad X_{m}^{\prime} \mapsto\left(1+q X_{i}^{-1}\right)^{-1} X_{m}
\end{gathered}
$$

(5) (the inverse of (4)) if $\lambda_{k}$ is identified with $\lambda_{m}$, and $\lambda_{j}$ is distinct from $\lambda_{l}$, then

$$
\begin{aligned}
X_{j}^{\prime} & \mapsto\left(1+q X_{i}\right) X_{j} \quad X_{l}^{\prime} \mapsto\left(1+q X_{i}\right) X_{l} \\
X_{k}^{\prime} & \mapsto\left(1+q X_{i}^{-1}\right)^{-1}\left(1+q^{3} X_{i}^{-1}\right)^{-1} X_{k}
\end{aligned}
$$

(6) if $\lambda_{j}$ is identified with $\lambda_{k}$, and $\lambda_{l}$ is identified with $\lambda_{m}$ (in which case $S$ is a 3 -times punctured sphere), then

$$
X_{j}^{\prime} \mapsto X_{i} X_{j} \quad X_{l}^{\prime} \mapsto X_{i} X_{l} ;
$$

(7) (the inverse of (6)) if $\lambda_{j}$ is identified with $\lambda_{m}$, and $\lambda_{k}$ is identified with $\lambda_{l}$ (in which case $S$ is a 3-times punctured sphere), then

$$
X_{j}^{\prime} \mapsto X_{i} X_{j} \quad X_{k}^{\prime} \mapsto X_{i} X_{k} ;
$$

(8) if $\lambda_{j}$ is identified with $\lambda_{l}$, and $\lambda_{k}$ is identified with $\lambda_{m}$ (in which case $S$ is a once punctured torus), then

$$
\begin{gathered}
X_{j}^{\prime} \mapsto\left(1+q X_{i}\right)\left(1+q^{3} X_{i}\right) X_{j} \\
X_{k}^{\prime} \mapsto\left(1+q X_{i}^{-1}\right)^{-1}\left(1+q^{3} X_{i}^{-1}\right)^{-1} X_{k}
\end{gathered}
$$

Recall that $\widehat{\mathcal{K}}_{\tau}^{q}$ is the fraction division algebra of $\mathcal{K}_{\tau}^{q}$. The algebraic isomorphism between $\widehat{\mathcal{K}}_{\tau}^{q}$ and $\widehat{\mathcal{K}}_{\tau^{\prime}}^{q}$ is defined in Definition 5 .

Lemma 16 Suppose that a decorated ideal triangulation $\tau^{\prime}$ is obtained from $\tau$ by a mark rotation $\rho_{\mu}$ for some $\mu \in\{1,2, \ldots, 2 m\}$. Let $\lambda$ be the common underlying 
ideal triangulation of $\tau$ and $\tau^{\prime}$. The following diagram is commutative if and only if $a=q^{-2}$.

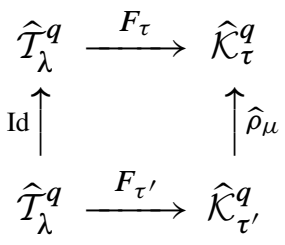

Proof It is enough to check $F_{\tau}\left(X_{i}\right)=\hat{\rho}_{\mu} \circ F_{\tau^{\prime}}\left(X_{i}\right)$ holds for any generator $X_{i}$.

If $\lambda_{i}$ does not bound a side of the ideal triangle $\tau_{\mu}$, then $F_{\tau}\left(X_{i}\right)=\hat{\rho}_{\mu} \circ F_{\tau^{\prime}}\left(X_{i}\right)$ holds automatically.

Suppose $\lambda_{i}$ bounds the $s$-side of $\tau_{\mu}$ and the $t$-side of $\tau_{\nu}$. If $\mu \neq v$, then $\lambda_{i}$ bounds the $(s+2)$ (modulo 3)-side of $\tau_{\mu}^{\prime}$ and the $t$-side of $\tau_{\nu}^{\prime}$. Then $F_{\tau}\left(X_{i}\right)=H_{\mu}^{s} H_{v}^{t}$ and $F_{\tau^{\prime}}\left(X_{i}\right)=H_{\mu}^{\prime s+2} H_{v}^{t}$. To show $F_{\tau}\left(X_{i}\right)=\hat{\rho}_{\mu} \circ F_{\tau^{\prime}}\left(X_{i}\right)$ is enough to show that $H_{\mu}^{s}=\hat{\rho}_{\mu}\left(H_{\mu}^{\prime s+2}\right)$.

If $\mu=v$, then then $\lambda_{i}$ bounds the $(s+2)$ (modulo 3)-side of $\tau_{\mu}^{\prime}$ and the $(t+2)$ (modulo 3)-side of $\tau_{\nu}^{\prime}$. Then $F_{\tau}\left(X_{i}\right)=q^{\sigma_{t s}} H_{\mu}^{s} H_{\mu}^{t}$ and $F_{\tau^{\prime}}\left(X_{i}\right)=q^{\sigma_{t s}} H_{\mu}^{\prime s+2} H_{\mu}^{\prime t+2}$. To show $F_{\tau}\left(X_{i}\right)=\hat{\rho}_{\mu} \circ F_{\tau^{\prime}}\left(X_{i}\right)$ is enough to show that $H_{\mu}^{s}=\hat{\rho}_{\mu}\left(H_{\mu}^{\prime s+2}\right)$ for $s \in\{0,1,2\}$, since $t \in\{0,1,2\}$.

When $s=0$, we have $H_{\mu}^{s}=Y_{\mu} Z_{\mu}^{-1}$ and $H_{\mu}^{s+2}=Y_{\mu}^{\prime-1}$. Now

$$
\begin{array}{rlrl}
H_{\mu}^{s} & =\hat{\rho}_{\mu}\left(H_{\mu}^{\prime s+2}\right) \\
& \Longleftrightarrow & Y_{\mu} Z_{\mu}^{-1} & =\hat{\rho}_{\mu}\left(Y_{\mu}^{\prime-1}\right) \\
& \Longleftrightarrow & Y_{\mu} Z_{\mu}^{-1} & =a^{-1} Z_{\mu}^{-1} Y_{\mu} \\
& & Z_{\mu} Y_{\mu} & =a^{-1} Y_{\mu} Z_{\mu} \\
& & a & =q^{-2} .
\end{array}
$$

When $s=1$, we have $H_{\mu}^{s}=Z_{\mu}$ and $H_{\mu}^{\prime s+2}=Y_{\mu}^{\prime} Z_{\mu}^{\prime-1}$. Now

$$
\begin{aligned}
H_{\mu}^{s} & =\hat{\rho}_{\mu}\left(H_{\mu}^{\prime s+2}\right) \\
Z_{\mu} & =\hat{\rho}_{\mu}\left(Y_{\mu}^{\prime} Z_{\mu}^{\prime-1}\right) \\
Z_{\mu} & =a Y_{\mu}^{-1} Z_{\mu} Y_{\mu} \\
Z_{\mu} & =a q^{2} Z_{\mu} \\
a & =q^{-2} .
\end{aligned}
$$


When $s=2$, we have $H_{\mu}^{s}=Y_{\mu}^{-1}$ and $H_{\mu}^{\prime s+2}=Z_{\mu}^{\prime}$. Now

\begin{tabular}{|c|c|}
\hline & $H_{\mu}^{i}=\hat{\rho}_{\mu}\left(H_{\mu}^{\prime i}\right.$ \\
\hline$\Longleftrightarrow$ & $Y_{\mu}^{-1}=\hat{\rho}_{\mu}\left(Z_{\mu}^{\prime}\right)$ \\
\hline & $Y_{\mu}^{-1}=Y_{\mu}^{-1}$ \\
\hline
\end{tabular}

This holds automatically.

Lemma 17 Suppose that a decorated ideal triangulation $\tau^{\prime}$ is obtained from $\tau$ by a diagonal exchange $\varphi_{\mu \nu}$. Let $\lambda$ and $\lambda^{\prime}$ be the underlying ideal triangulation of $\tau$ and $\tau^{\prime}$ respectively. Then $\lambda^{\prime}$ is obtained $\lambda$ by a diagonal exchange with respect to the edge $\lambda_{i}$ which is the common edge of $\tau_{\mu}$ and $\tau_{\nu}$. The following diagram is commutative if and only if $b=q^{3}$.
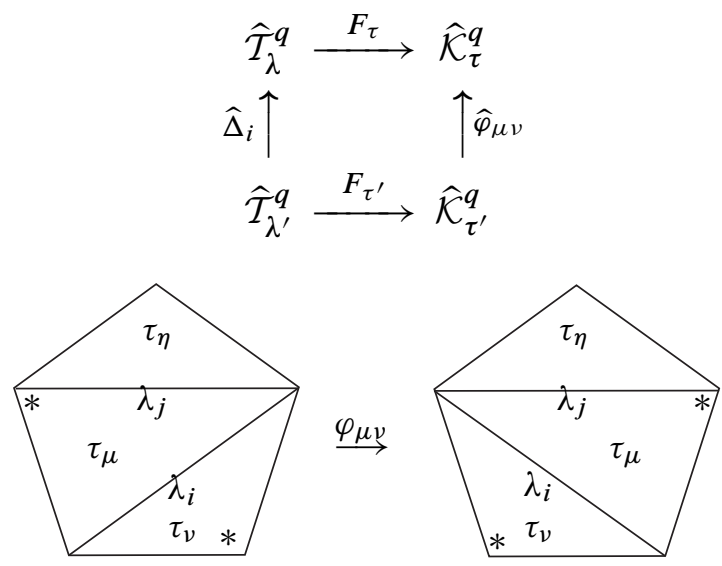

Figure 5

Proof First we show that $b=q^{3}$ is necessary. As in Figure 5, $\mu, v, \eta$ are different. We have $F_{\tau}\left(X_{i}\right)=Y_{\mu} Z_{\mu}^{-1} Y_{\nu} Z_{\nu}^{-1}$ and $F_{\tau}\left(X_{j}\right)=H_{\eta}^{s} Z_{\mu}$, for some $s \in\{0,1,2\}$. And $F_{\tau^{\prime}}\left(X_{j}\right)=H_{\eta}^{j} Y_{\mu}^{\prime-1}$. That the diagram is commutative implies

$$
\begin{array}{rlrl}
\hat{\varphi}_{\mu \nu} \circ F_{\tau^{\prime}}\left(X_{j}\right) & =F_{\tau} \circ \widehat{\Delta}_{i}\left(X_{j}^{\prime}\right) \\
\hat{\varphi}_{\mu \nu}\left(H_{\eta}^{j} Y_{\mu}^{\prime-1}\right) & =F_{\tau}\left(\left(1+q X_{i}\right) X_{j}\right) \\
& \Longleftrightarrow \quad H_{\eta}^{s}\left[\left(b Y_{\mu} Y_{v}+Z_{\mu} Z_{v}\right)^{-1} Z_{\nu}\right]^{-1} & =\left(1+q Y_{\mu} Z_{\mu}^{-1} Y_{\nu} Z_{\nu}^{-1}\right) H_{\eta}^{s} Z_{\mu} \\
& \Longleftrightarrow \quad b Y_{\mu} Y_{\nu}+Z_{\mu} Z_{v} & =Z_{\nu}\left(1+q Y_{\mu} Z_{\mu}^{-1} Y_{\nu} Z_{\nu}^{-1}\right) Z_{\mu} \\
& =Z_{\mu} Z_{v}+q^{3} Y_{\mu} Y_{\nu} \\
& \Longleftrightarrow \quad b & =q^{3} .
\end{array}
$$


In the following we show $b=q^{3}$ is also sufficient. There are eight cases in Proposition 15 to check. For instance, we verify Case 4 . By definition, We have

$$
\begin{array}{ll}
F_{\tau}\left(X_{i}\right)=Y_{\mu} Z_{\mu}^{-1} Y_{\nu} Z_{v}^{-1} & F_{\tau^{\prime}}\left(X_{i}\right)=Y_{\mu}^{\prime} Z_{\mu}^{\prime-1} Y_{\nu}^{\prime} Z_{v}^{\prime-1} \\
F_{\tau}\left(X_{j}\right)=Z_{\mu} Z_{v} & F_{\tau^{\prime}}\left(X_{j}\right)=Y_{\mu}^{\prime-1} Y_{v}^{\prime-1} \\
F_{\tau}\left(X_{k}\right)=Y_{v}^{-1} H_{\zeta}^{s} & F_{\tau^{\prime}}\left(X_{k}\right)=Z_{\mu}^{\prime} H_{\zeta}^{s} \\
F_{\tau}\left(X_{m}\right)=Y_{\mu}^{-1} H_{\eta}^{t} & F_{\tau^{\prime}}\left(X_{m}\right)=Z_{v}^{\prime} H_{\eta}^{t}
\end{array}
$$

for some $s, t \in\{0,1,2\}$.

Then we have

$$
\begin{aligned}
& \hat{\varphi}_{\mu \nu} \circ F_{\tau^{\prime}}\left(X_{i}\right)=\hat{\varphi}_{\mu \nu}\left(Y_{\mu}^{\prime} Z_{\mu}^{\prime-1} Y_{\nu}^{\prime} Z_{\nu}^{\prime-1}\right) \\
& =\left(b Y_{\mu} Y_{\nu}+Z_{\mu} Z_{v}\right)^{-1} Z_{\nu} b^{-1} Y_{\mu}^{-1}\left(b Y_{\mu} Y_{\nu}+Z_{\mu} Z_{v}\right) \\
& \left(b Y_{\mu} Y_{\nu}+Z_{\mu} Z_{v}\right)^{-1} Z_{\mu} b^{-1} Y_{v}^{-1}\left(b Y_{\mu} Y_{\nu}+Z_{\mu} Z_{v}\right) \\
& =b^{-2}\left(b Y_{\mu} Y_{\nu}+Z_{\mu} Z_{v}\right)^{-1} Z_{\nu} Y_{\mu}^{-1} Z_{\mu} Y_{\nu}^{-1}\left(b Y_{\mu} Y_{\nu}+Z_{\mu} Z_{\nu}\right) \\
& =b^{-2}\left(b Y_{\mu} Y_{\nu}+Z_{\mu} Z_{v}\right)^{-1}\left(b q^{4} Y_{\mu} Y_{v}+q^{4} Z_{\mu} Z_{v}\right) Z_{\nu} Y_{\mu}^{-1} Z_{\mu} Y_{v}^{-1} \\
& =b^{-2} q^{4} Z_{v} Y_{\mu}^{-1} Z_{\mu} Y_{v}^{-1} \\
& =b^{-2} q^{6} Z_{\nu} Y_{v}^{-1} Z_{\mu} Y_{\mu}^{-1} \\
& =b^{-2} q^{6} F_{\tau}\left(X_{i}^{-1}\right) \\
& =b^{-2} q^{6} F_{\tau} \circ \widehat{\Delta}_{i}\left(X_{i}\right) \\
& =F_{\tau} \circ \widehat{\Delta}_{i}\left(X_{i}\right)
\end{aligned}
$$

due to the assumption that $b=q^{3}$.

And

$$
\begin{aligned}
& \hat{\varphi}_{\mu \nu} \circ F_{\tau^{\prime}}\left(X_{j}\right)=\hat{\varphi}_{\mu \nu}\left(Y_{\mu}^{\prime-1} Y_{\nu}^{\prime-1}\right) \\
& =Z_{v}^{-1}\left(b Y_{\mu} Y_{v}+Z_{\mu} Z_{v}\right) Z_{\mu}^{-1}\left(b Y_{\mu} Y_{v}+Z_{\mu} Z_{v}\right) \\
& =\left(b Z_{v}^{-1} Y_{\mu} Y_{\nu} Z_{\mu}^{-1}+1\right)\left(b Y_{\mu} Y_{\nu}+Z_{\mu} Z_{\nu}\right) \\
& =\left(b q^{-2} Y_{\mu} Y_{\nu} Z_{v}^{-1} Z_{\mu}^{-1}+1\right)\left(b Y_{\mu} Y_{\nu} Z_{v}^{-1} Z_{\mu}^{-1}+1\right) Z_{\mu} Z_{v} \\
& =\left(q Y_{\mu} Y_{\nu} Z_{\nu}^{-1} Z_{\mu}^{-1}+1\right)\left(q^{3} Y_{\mu} Y_{\nu} Z_{v}^{-1} Z_{\mu}^{-1}+1\right) Z_{\mu} Z_{v} \\
& =\left(1+q F_{\tau}\left(X_{i}\right)\right)\left(1+q^{3} F_{\tau}\left(X_{i}\right)\right) F_{\tau}\left(X_{j}\right) \\
& =F_{\tau}\left(\left(1+q X_{i}\right)\left(1+q^{3} X_{i}\right) X_{j}\right) \\
& =F_{\tau} \circ \widehat{\Delta}_{i}\left(X_{j}\right) \text {. }
\end{aligned}
$$


And

$$
\begin{aligned}
\hat{\varphi}_{\mu \nu} \circ F_{\tau^{\prime}}\left(X_{k}\right) & =\hat{\varphi}_{\mu \nu}\left(Z_{\mu}^{\prime}\right) H^{s} \\
& =b\left(b Y_{\mu} Y_{\nu}+Z_{\mu} Z_{v}\right)^{-1} Y_{\mu} H_{\zeta}^{s} \\
& =b\left[Y_{\mu} Y_{v}\left(b+Y_{\mu}^{-1} Y_{v}^{-1} Z_{\mu} Z_{v}\right)\right]^{-1} Y_{\mu} H_{\zeta}^{s} \\
& =b\left(b+Y_{\mu}^{-1} Y_{v}^{-1} Z_{\mu} Z_{v}\right)^{-1} Y_{v}^{-1} H_{\zeta}^{s} \\
& =b\left(b+q^{4} Z_{\mu} Z_{v} Y_{\mu}^{-1} Y_{v}^{-1}\right)^{-1} Y_{v}^{-1} H_{\zeta}^{s} \\
& =\left(1+q Z_{\mu} Z_{v} Y_{\mu}^{-1} Y_{v}^{-1}\right)^{-1} Y_{v}^{-1} H_{\zeta}^{s} \\
& =\left(1+q F_{\tau}\left(X_{i}\right)^{-1}\right) F_{\tau}\left(X_{k}\right) \\
& =F_{\tau}\left(\left(1+q X_{i}^{-1}\right) X_{k}\right) \\
& =F_{\tau} \circ \widehat{\Delta}_{i}\left(X_{k}\right) .
\end{aligned}
$$

It is same for $X_{m}^{\prime}$ due to the symmetry of $\mu, \nu$.

Theorem 18 Suppose the decorated ideal triangulations $\tau$ and $\tau^{\prime}$ have the underlying ideal triangulations $\lambda$ and $\lambda^{\prime}$ respectively. The following diagram is commutative if and only if $a=q^{-2}, b=q^{3}$.

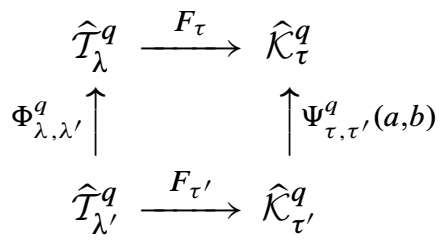

Proof By Theorem 2, $\tau$ and $\tau^{\prime}$ are connected by a sequence $\tau=\tau_{(0)}, \tau_{(1)}, \ldots$, $\tau_{(n)}=\tau^{\prime}$ where each $\tau_{(k+1)}$ is obtained from $\tau_{(k)}$ by a reindexing or a mark rotation or a diagonal exchange. For a reindexing, the diagram is always commutative. By Lemmas 16 and 17, the the diagram is always commutative if and only if $a=q^{-2}$ and $b=q^{3}$.

Recall that the quantum Teichmüller space of $S$ is defined as the algebra

$$
\hat{\mathcal{T}}_{S}^{q}=\left(\bigsqcup_{\lambda \in \Lambda(S)} \hat{\mathcal{T}}_{\lambda}^{q}\right) / \sim
$$

where the relation $\sim$ is defined by the property that, for $X \in \hat{\mathcal{T}}_{\lambda}^{q}$ and $X^{\prime} \in \hat{\mathcal{T}}_{\lambda^{\prime}}^{q}$,

$$
X \sim X^{\prime} \Longleftrightarrow X=\Phi_{\lambda, \lambda^{\prime}}^{q}\left(X^{\prime}\right) .
$$


And the generalized Kashaev algebra $\widehat{\mathcal{K}}_{S}^{q}(a, b)$ associated to a surface $S$ is defined as the algebra

$$
\widehat{\mathcal{K}}_{S}^{q}(a, b)=\left(\bigsqcup_{\tau \in \Delta(S)} \hat{\mathcal{K}}_{\tau}^{q}(a, b)\right) / \sim
$$

where the relation $\sim$ is defined by the property that, for $X \in \widehat{\mathcal{K}}_{\tau}^{q}(a, b)$ and $X^{\prime} \in$ $\widehat{\mathcal{K}}_{\tau^{\prime}}^{q}(a, b)$,

$$
X \sim X^{\prime} \Leftrightarrow X=\Psi_{\tau, \tau^{\prime}}^{q}(a, b)\left(X^{\prime}\right)
$$

Corollary 19 The homomorphism $F_{\tau}$ induces a homomorphism $\hat{\mathcal{T}}_{S}^{q} \rightarrow \widehat{\mathcal{K}}_{S}^{q}(a, b)$ if and only if $a=q^{-2}, b=q^{3}$.

\subsection{Quotient algebra}

Furthermore, consider the element

$$
H=q^{-\sum_{i<j} \sigma_{i j}^{\lambda}} X_{1} X_{2} \ldots X_{3 m} \in \mathcal{T}_{\lambda}^{q} .
$$

It is proved in $[14$, Proposition 4$]$ that $H$ is independent of the ideal triangulation $\lambda$. Therefore $H$ is a well-defined element of the quantum Teichmüller space $\widehat{\mathcal{T}}_{S}^{q}$.

Theorem 20 The homomorphism $F_{\tau}$ induces a homomorphism

$$
\hat{\mathcal{T}}_{S}^{q} /\left(q^{-2 m} H\right) \rightarrow \hat{\mathcal{K}}_{S}^{q}\left(q^{-2}, q^{3}\right)
$$

where $\left(q^{-2 m} H\right)$ is the ideal generated by $q^{-2 m} H$.

Proof We only need to show that $F_{\tau}\left(q^{-2 m} H\right)=1$ for any arbitrary decorated ideal triangulation $\tau$. In fact

$$
F_{\tau}\left(X_{1} X_{2} \ldots X_{3 m}\right)=q^{\delta_{\mu_{1} \nu_{1}}} H_{\mu_{1}}^{s_{1}} H_{v_{1}}^{t_{1}} \ldots q^{\delta_{\mu_{3 m} \nu_{3 m}}} H_{\mu_{3 m}}^{s_{3 m}} H_{\nu_{3 m}}^{t_{3 m}} .
$$

where the edge $\lambda_{i}$ bounds the $s_{i}$-side of $\tau_{\mu_{i}}$ and the $t_{i}$-side of $\tau_{\nu_{i}}$ for $i=1, \ldots, 3 m$. Since $H_{\mu}^{s}$ and $H_{v}^{t}$ are commutative when $\mu \neq v$, we may collect the terms indexed by the same ideal triangle by commutating the terms indexed by different ideal triangles. The right hand side of the above identity is equal to

$$
\prod_{\mu=1}^{2 m} P_{\mu}
$$

where $P_{\mu}$ is the product of terms involving the ideal triangle $\tau_{\mu}$.

Case 1 If $\tau_{\mu}$ is embedded, then $P_{\mu}=H_{\mu}^{r} H_{\mu}^{s} H_{\mu}^{t}$, where $\{r, s, t\}=\{0,1,2\}$. 
When $(r, s, t)$ is an even permutation of $0,1,2$, we have $P_{\mu}=1$.

Suppose the $r$-side, the $s$-side and the $t$-side of $\tau_{\mu}$ are bounded by edges $\lambda_{i}, \lambda_{j}$ and $\lambda_{k}$ respectively. Then $i \leq j \leq k$ since this order is preserved when we commutate the terms indexed by different ideal triangles. Denote by $\sigma_{i j}^{\mu}$ the number of corners of $\tau_{\mu}$ delimited by $\lambda_{i}$ from the left and delimited by $\lambda_{j}$ from the right minus the number of corners of $\tau_{\mu}$ delimited by $\lambda_{j}$ from the left and delimited by $\lambda_{i}$ from the right. Then

$$
\sigma_{i j}^{\mu}+\sigma_{j k}^{\mu}+\sigma_{i k}^{\mu}=-1-1+1=-1 .
$$

Therefore

$$
P_{\mu}=1=q^{1+\sigma_{i j}^{\mu}+\sigma_{j k}^{\mu}+\sigma_{i k}^{\mu}} .
$$

When $(r, s, t)$ is an odd permutation of $0,1,2$, we have $P_{\mu}=q^{2}$. And

$$
\sigma_{i j}^{\mu}+\sigma_{j k}^{\mu}+\sigma_{i k}^{\mu}=1+1-1=1 .
$$

Therefore

$$
P_{\mu}=q^{2}=q^{1+\sigma_{i j}^{\mu}+\sigma_{j k}^{\mu}+\sigma_{i k}^{\mu}} .
$$

Case 2 If $\tau_{\mu}$ is not embedded, then $P_{\mu}=q^{\sigma_{s r}} H_{\mu}^{r} H_{\mu}^{s} H_{\mu}^{t}$ or $P_{\mu}=q^{\sigma_{t s}} H_{\mu}^{r} H_{\mu}^{s} H_{\mu}^{t}$. When $(r, s, t)$ is an even permutation of $0,1,2$, we have $P_{\mu}=q \cdot 1=q$. When $(r, s, t)$ is an odd permutation of $0,1,2$, we have $P_{\mu}=q^{-1} \cdot q^{2}=q$. And we always have

$$
\sigma_{i j}^{\mu}+\sigma_{j k}^{\mu}+\sigma_{i k}^{\mu}=0 \text {. }
$$

Therefore

$$
P_{\mu}=q=q^{1+\sigma_{i j}^{\mu}+\sigma_{j k}^{\mu}+\sigma_{i k}^{\mu}} .
$$

Combining the two cases, we obtain

$$
F_{\tau}\left(X_{1} X_{2} \ldots X_{3 m}\right)=\prod_{\mu=1}^{2 m} P_{\mu}=\prod_{\mu=1}^{2 m} q^{1+\sigma_{i j}^{\mu}+\sigma_{j k}^{\mu}+\sigma_{i k}^{\mu}}=q^{2 m+\sum_{i<j} \sigma_{i j}^{\lambda}} .
$$

Thus $F_{\tau}\left(q^{-2 m} H\right)=1$.

\section{Appendix A Kashaev coordinates and Penner coordinates}

We review the relationship of Kasheev coordinate and Penner coordinates following Kashaev [9] and Teschner [16].

A decorated hyperbolic metric $(d, r)$ on $S$, introduced by Penner [15], is a complete hyperbolic metric $d$ so that each end is cusp type and each cusp $c_{i}$ is assigned a positive number $r_{i}$. The decorated Teichmüller space is the space of isotopy class of 
decorated hyperbolic metrics. For each decorated hyperbolic metric $(d, r)$, at each $\operatorname{cusp} c_{i}$, there is a horocycle with boundary length $r_{i}$. Under a decorated hyperbolic metric, each edge of an ideal triangulation of a punctured surface $S$ is realized as a geodesic running from one puncture to another. Penner coordinate $\delta(e)$ at an edge $e$ is the signed distance between two horocycles bounding cusps $c_{i}$ and $c_{j}$ if the edge $e$ runs from $c_{i}$ to $c_{j}$. Denote by $\overline{\mathcal{T}}_{\lambda}$ the decorated Teichmüller space parameterized by Penner coordinates associated to the ideal triangulation $\lambda$.

Let $\tau$ be a decorated ideal triangulation with the underlying ideal triangulation $\lambda$. Let $\mathcal{K}_{\tau}=\mathbb{R}^{4 m}=\left\{\left(\ln y_{1}, \ln z_{1}, \ldots, \ln y_{2 m}, \ln z_{2 m}\right)\right\}$ be the space of Kashaev coordinates. There is a map $f: \overline{\mathcal{T}}_{\lambda} \rightarrow \mathcal{K}_{\tau}$ defined as follows.

For an ideal triangle $\tau_{i}$ (embedded or not) with a marked corner, there are three sides which correspond to the three half-edges incident to the vertex $\tau_{\mu}^{*}$ of the dual graph. The three sides are numerated by $0,1,2$ in the counterclockwise order such that the $0-$ side is opposite to the marked corner. Denote by $\lambda_{i}^{0}, \lambda_{i}^{1}, \lambda_{i}^{2}$ the edges (two of them may coincide) bounding the three sides of $\tau_{i}$. We define

$$
y_{i}=e^{\frac{1}{2}\left(\delta\left(\lambda_{1}^{i}\right)-\delta\left(\lambda_{0}^{i}\right)\right)}, \quad z_{i}=e^{\frac{1}{2}\left(\delta\left(\lambda_{2}^{i}\right)-\delta\left(\lambda_{0}^{i}\right)\right)} .
$$

Proposition 21 (Kashaev [9]) The following sequence is exact:

$$
1 \longrightarrow \mathbb{R}_{+} \longrightarrow \overline{\mathcal{T}}_{\lambda} \stackrel{f}{\rightarrow} \mathcal{K}_{\tau} \longrightarrow H^{1}(S, \mathbb{R}) \longrightarrow 0 .
$$

Proposition 22 (Kashaev [9]) If

$$
\left(\ln y_{1}, \ln z_{1}, \ldots, \ln y_{2 m}, \ln z_{2 m}\right)=f\left(\delta\left(\lambda_{1}\right), \ldots, \delta\left(\lambda_{3 m}\right)\right),
$$

then the two 2-forms are equal:

$\sum_{\mu_{1}}^{2 m} d \ln y_{\mu} \wedge d \ln z_{\mu}=f^{*}\left(\sum_{\mu=1}^{2 m} d \delta\left(\lambda_{i}\right) \wedge d \delta\left(\lambda_{j}\right)+d \delta\left(\lambda_{j}\right) \wedge d \delta\left(\lambda_{k}\right)+d \delta\left(\lambda_{k}\right) \wedge d \delta\left(\lambda_{i}\right)\right)$ where $\lambda_{i}, \lambda_{j}, \lambda_{k}$ are edges bounding the three sides of $\tau_{\mu}$ in the counterclockwise order.

Proposition 23 (Kashaev [9]) Suppose that the decorated ideal triangulations $\tau$ and $\tau^{\prime}$ have the underlying ideal triangulations $\lambda$ and $\lambda^{\prime}$ respectively. The following diagram is commutative:

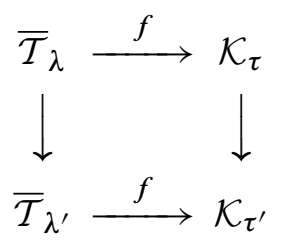


where the two vertical maps are corresponding coordinate changes. The coordinate changes of Kashaev coordinates are given in Definition 4.

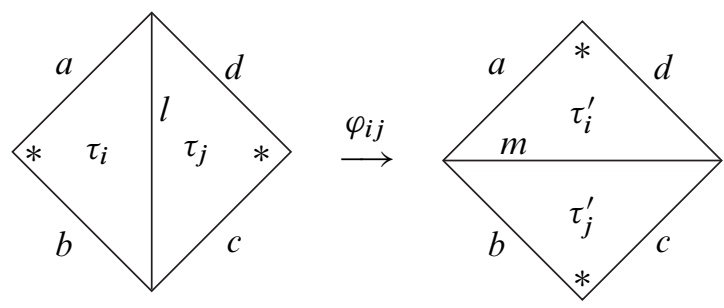

Figure 6

Proof For a reindexing, the conclusion is obvious. For a mark rotation, the conclusion is easily proved by applying the definition of $\left(y_{i}, z_{i}\right)$. For a diagonal exchange, we need to use the famous Ptolemy relation for Penner coordinates.

In Figure 6 , denote by $a, b, c, d, l$ and $m$ the Penner coordinates of the corresponding edges. If the ideal triangles are not embedded, some of the numbers $a, b, c, d$ may equal. The Ptolemy relation is

$$
e^{\frac{1}{2}(l+m)}=e^{\frac{1}{2}(a+c)}+e^{\frac{1}{2}(b+d)}
$$

which holds in spite of whether the ideal triangles $\tau_{i}, \tau_{j}$ are embedded or not.

We show the relation between $\left(y_{i}, z_{i}, y_{j}, z_{j}\right)$ and $\left(y_{i}^{\prime}, z_{i}^{\prime}, y_{j}^{\prime}, z_{j}^{\prime}\right)$ in Definition 4 holds. In fact,

$$
\begin{aligned}
\frac{z_{j}}{y_{i} y_{j}+z_{i} z_{j}} & =\frac{e^{\frac{1}{2}(d-l)}}{e^{\frac{1}{2}(a-l)} e^{\frac{1}{2}(c-l)}+e^{\frac{1}{2}(b-l)} e^{\frac{1}{2}(d-l)}} \\
& =\frac{e^{\frac{1}{2}(d+l)}}{e^{\frac{1}{2}(a+c)}+e^{\frac{1}{2}(b+d)}} \\
& =\frac{e^{\frac{1}{2}(d+l)}}{e^{\frac{1}{2}(l+m)}} \\
& =e^{\frac{1}{2}(d-m)} \\
& =y_{i}^{\prime} .
\end{aligned}
$$

The same calculation can be used to verify the formula of $z_{i}^{\prime}, y_{j}^{\prime}, z_{j}^{\prime}$. 


\section{Acknowledgments}

The authors would like to thank Francis Bonahon and Feng Luo for encouragement and helpful comments, Liang Kong and Hua Bai for helpful discussion. A part of the work of this paper was done when the second author was visiting Chern Institute of Mathematics, Tianjin, China. He would like to take the opportunity to thank Chern Institute for hospitality. The authors thank the referee for valuable suggestion.

\section{References}

[1] H Bai, A uniqueness property for the quantization of Teichmüller spaces, Geom. Dedicata 128 (2007) 1-16 MR2350143

[2] H Bai, F Bonahon, X Liu, Local representations of the quantum Teichmüller space arXiv:0707.2151

[3] F Bonahon, Shearing hyperbolic surfaces, bending pleated surfaces and Thurston's symplectic form, Ann. Fac. Sci. Toulouse Math. (6) 5 (1996) 233-297 MR1413855

[4] F Bonahon, Quantum Teichmüller theory and representations of the pure braid group, Commun. Contemp. Math. 10 (2008) 913-925 MR2468371

[5] F Bonahon, X Liu, Representations of the quantum Teichmüller space and invariants of surface diffeomorphisms, Geom. Topol. 11 (2007) 889-937 MR2326938

[6] L O Chekhov, V V Fock, Observables in 3D gravity and geodesic algebras, Czechoslovak J. Phys. 50 (2000) 1201-1208 MR1806262 Quantum groups and integrable systems (Prague, 2000)

[7] V V Fock, Dual Teichmüller spaces arXiv:dg-ga/9702018

[8] V V Fok, L O Chekhov, Quantum Teichmüller spaces, Teoret. Mat. Fiz. 120 (1999) 511-528 MR1737362

[9] R M Kashaev, Quantization of Teichmüller spaces and the quantum dilogarithm, Lett. Math. Phys. 43 (1998) 105-115 MR1607296

[10] R M Kashaev, The Liouville central charge in quantum Teichmüller theory, Tr. Mat. Inst. Steklova 226 (1999) 72-81 MR1782553

[11] R M Kashaev, On the spectrum of Dehn twists in quantum Teichmüller theory, from: "Physics and combinatorics, 2000 (Nagoya)", World Sci. Publ., River Edge, NJ (2001) 63-81 MR1872252

[12] R M Kashaev, On quantum moduli space of flat $\mathrm{PSL}_{2}(\mathbb{R})$-connections on a punctured surface, from: "Handbook of Teichmüller theory Vol I", IRMA Lect. Math. Theor. Phys. 11, Eur. Math. Soc., Zürich (2007) 761-782 MR2349684 
[13] X Liu, Gromov-Witten invariants and moduli spaces of curves, from: "International Congress of Mathematicians Vol II”, Eur. Math. Soc., Zürich (2006) 791-812 MR2275623

[14] X Liu, The quantum Teichmüller space as a non-commutative algebraic object, J. Knot Theory Ramifications 18 (2009) 705-726

[15] R C Penner, The decorated Teichmüller space of punctured surfaces, Comm. Math. Phys. 113 (1987) 299-339 MR919235

[16] J Teschner, An analog of a modular functor from quantized Teichmüller theory, from: "Handbook of Teichmüller theory Vol I", IRMA Lect. Math. Theor. Phys. 11, Eur. Math. Soc., Zürich (2007) 685-760 MR2349683

[17] W P Thurston, The topology and geometry of 3-manifolds, lecture notes, Princeton University (1976-1979) Available at http://msri.org/communications/books/ gt $3 \mathrm{~m}$

School of Mathematics, University of Minnesota Minneapolis, MN 55455, USA

Department of Mathematics, Columbia University 2990 Broadway, New York, NY 10027, USA

guoxx170@math.umn.edu, xiaoboli@math.columbia.edu

Received: 6 May 2009 